\title{
Distribution of orbits of geometrically finite groups acting on null vectors
}

\author{
Nattalie Tamam ${ }^{1} \cdot$ Jacqueline M. Warren $^{1}$ (D) \\ Received: 12 October 2020 / Accepted: 9 December 2021 / Published online: 27 January 2022 \\ (c) The Author(s) 2022
}

\begin{abstract}
We study the distribution of non-discrete orbits of geometrically finite groups in $\operatorname{SO}(n, 1)$ acting on $\mathbb{R}^{n+1}$, and more generally on the quotient of $\mathrm{SO}(n, 1)$ by a horospherical subgroup. Using equidistribution of horospherical flows, we obtain both asymptotics for the distribution of orbits for the action of general geometrically finite groups, and we obtain quantitative statements with additional assumptions.
\end{abstract}

Keywords Homogeneous dynamics · Ergodic theory · Discrete subgroups of Lie groups · Flows in homogeneous spaces

Mathematics Subject Classification 22F30 $\cdot 22 \mathrm{E} 40 \cdot 37 \mathrm{~A} 05$

\section{Contents}

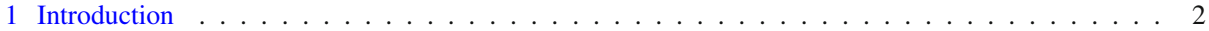

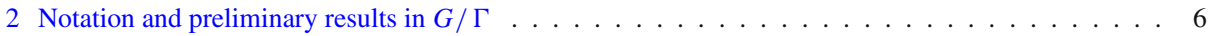

2.1 Thick-thin decomposition and the shadow lemma . . . . . . . . . . . . . . . . . 7

2.2 Patterson-Sullivan measure . . . . . . . . . . . . . . . . . . . . . . . . . 8

2.3 Burger-Roblin and Bowen-Margulis-Sullivan measures . . . . . . . . . . . . . . . . . . . . 14

2.4 Sobolev norms . . . . . . . . . . . . . . . . . . . . . . . . . . 15

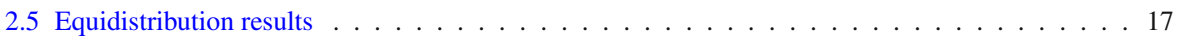

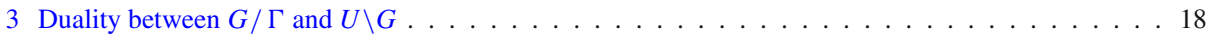

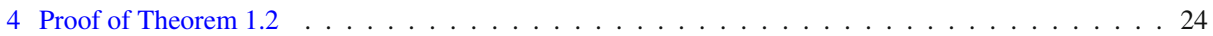

5 A small support "Ergodic Theorem" . . . . . . . . . . . . . . . . . . . . . . . 27

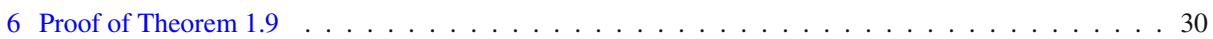

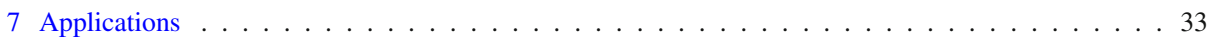

7.1 Identification with null vectors . . . . . . . . . . . . . . . . . . . 35

7.2 Wedge products . . . . . . . . . . . . . . . . . . . 36

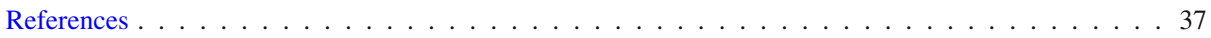

$凶$ Jacqueline M. Warren

j4warren@ucsd.edu

1 Department of Mathematics, University of California, San Diego, USA 


\section{Introduction}

We often seek to understand a group through the distribution of its orbits on a given space. In this paper, we will consider the action of certain geometrically finite groups on $\mathbb{R}^{n+1}$ and other spaces.

When $\Gamma$ is a lattice in $\mathrm{SL}_{2}(\mathbb{R})$ acting on $\mathbb{R}^{2}$, this question was considered by Ledrappier [10], who proved that

$$
\lim _{T \rightarrow \infty} \frac{1}{T} \sum_{\gamma \in \Gamma,\|\gamma\| \leq T} f(X \gamma)=c(\Gamma) \int_{\mathbb{R}^{2}} \frac{f(Y)}{|X||Y|} d Y
$$

for compactly supported functions $f$ and $X \in \mathbb{R}^{2}$, where $c(\Gamma)$ is some constant depending on the covolume of the lattice $\Gamma$, and $\|\gamma\|$ denotes the $\ell_{2}$ norm on $\Gamma$. Nogueira [15] independently obtained this result for $\Gamma=\mathrm{SL}_{2}(\mathbb{Z})$ using different methods. More recently, Macourant and Weiss obtained a quantitative version of this theorem for cocompact lattices in $\mathrm{SL}_{2}(\mathbb{R})$, and also for $\Gamma=\mathrm{SL}_{2}(\mathbb{Z})$ in [13]. The case of lattices in $\mathrm{SL}_{n}(\mathbb{R})$ acting on different spaces $V$ has also been considered, see for instance [5,7].

In [16], Pollicott proved a similar quantitative theorem for the action of a lattice in $\mathrm{SL}_{2}(\mathbb{C})$ on $\mathbb{C}^{2}$. In the $p$-adic case, Ledrappier and Pollicott [11] considered lattices in $\mathrm{SL}_{2}\left(\mathbb{Q}_{p}\right)$ acting on $\mathbb{Q}_{p}^{2}$.

Similar questions have been studied extensively for lattices in a wide variety of groups $G$. For instance, Gorodnik and Weiss consider in [8] second countable, locally compact groups $G$ with a general axiomatic approach, with several examples. More recently, Gorodnik and Nevo comprehensively studied the action of a lattice in a connected algebraic Lie group acting on infinite volume homogeneous varieties in [6], including obtaining quantitative results under appropriate assumptions.

The case when $\Gamma$ has infinite covolume was recently studied by Maucourant and Schapira in [12], where they obtained an asymptotic version of Ledrappier's result for convex cocompact subgroups of $\mathrm{SL}_{2}(\mathbb{R})$, with a scaling factor permitted. Moreover, they prove that an ergodic theorem like Ledrappier's in the lattice case cannot be obtained in the infinite volume setting, because there is not even a ratio ergodic theorem. More specifically, [12, Proposition 1.5] shows that if $\Gamma \subseteq \mathrm{SL}_{2}(\mathbb{R})$ is geometrically finite with $-I$ the unique torsion element, then there exist small bump functions $f$ and $g$ such that for $\bar{v}$-almost every $v$ (where $\bar{v}$ is defined in Sect. 7),

$$
\frac{\sum_{\gamma \in \Gamma_{T}} f(v \gamma)}{\sum_{\gamma \in \Gamma_{T}} g(v \gamma)}
$$

does not have a limit. Thus, it is impossible to obtain an ergodic theorem in this setting with a normalization factor that does not depend on the functions. The key obstruction is the fluctuating behaviour of the Patterson-Sullivan measure. However, they show that with an additional averaging to address these fluctuations, there is a Log-Cesaro convergence, see [12, Theorem 1.6].

Throughout this paper, let $G=\mathrm{SO}(n, 1)^{\circ}$ and let $\Gamma \subseteq G$ be a Zariski dense geometrically finite subgroup. As a consequence of a more general ratio theorem we will discuss later in this section, we will obtain the following asymptotic behaviour for $\Gamma$ orbits acting on

$$
V=\mathbf{e}_{n+1} G \backslash\{0\},
$$


which is similar to a result of Maucourant and Schapira for $n=2$. Note that $V$ consists of null vectors of a certain quadratic form and corresponds to the upper half of the "light cone" in the usual representation of $\mathrm{SO}(n, 1)$; see Sect. 7.1 for more details.

When $\Gamma$ is geometrically finite, the limit set of $\Gamma$, denoted $\Lambda(\Gamma) \subseteq \partial\left(\mathbb{H}^{n}\right)$, decomposes into radial and bounded parabolic limit points:

$$
\Lambda(\Gamma)=\Lambda_{r}(\Gamma) \sqcup \Lambda_{b p}(\Gamma) .
$$

For the precise definitions, see Sect. 2.

Proposition 1.1 Let $\Gamma$ be convex cocompact. For any $\bar{\varphi} \in C_{c}(V)$ and every $v \in V$ with $v^{-} \in \Lambda(\Gamma)$, as $T \rightarrow \infty$, we have that

$$
\frac{1}{T^{\delta_{\Gamma} / 2}} \sum_{\gamma \in \Gamma_{T}} \bar{\varphi}(v \gamma) \asymp \int_{V} \bar{\varphi}(u) \frac{d \bar{\nu}(u)}{\left(\|v\|_{2}\|u\|_{2}\right)^{\delta_{\Gamma} / 2}},
$$

where the implied constant depends on $v$ and $\Gamma$. Here, $\delta_{\Gamma}$ denotes the critical exponent of $\Gamma$, $\|u\|_{2}$ denotes the Euclidean norm of $u \in \mathbb{R}^{n+1}$, and $\Gamma_{T}=\{\gamma \in \Gamma:\|\gamma\| \leq T\}$, where $\|\gamma\|$ denotes the max norm of $\gamma$ as a matrix in $\mathrm{SL}_{n+1}(\mathbb{R})$. The notation $v^{-} \in \Lambda_{r}(\Gamma)$ is discussed in Sect. 7.

Here, the notation $a \asymp b$ means that there exists a constant $\lambda>1$ such that

$$
\lambda^{-1} \leq \frac{a}{b} \leq \lambda .
$$

The precise definition of the measure $\bar{v}$ is discussed in Sect. 7. It is the pushforward of the measure $v$ defined in Sect. 2.3, which is part of the product structure of the Burger-Roblin $(B R)$ measure, defined fully in that section.

Let $U=\left\{u_{\mathbf{t}}: \mathbf{t} \in \mathbb{R}^{n-1}\right\}$ be the expanding horospherical subgroup for the frame flow $A$. Let $P \subset G$ be the parabolic subgroup which contains the contracting horospherical subgroup. Parametrizations of these groups are given in Sect. 2.

Proposition 1.1 is obtained by counting orbit points in $U \backslash G$. We will also establish a stronger version, specifically showing that a more precise ratio tends to 1 . With additional assumptions on $\Gamma$, we obtain a quantitative version of this statement. We need to define additional notation in order to state this result.

Let $U A K$ be the Iwasawa decomposition of $\operatorname{SL}_{n+1}(\mathbb{R})$, and let $\Psi: U \backslash G \rightarrow G$ be the map

$$
\Psi(U g)=a k
$$

where $g=u a k$ in the Iwasawa decomposition.

We view $G$ as embedded in $\operatorname{SL}_{n+1}(\mathbb{R})$. For $g \in G$, let $\|g\|$ denote the max norm as a matrix in $\mathrm{SL}_{n+1}(\mathbb{R})$. The following "product" is useful for our statements (a similar definition exists in the $\mathrm{SL}_{2}(\mathbb{R})$ case). For $x, y \in U \backslash G$, let

$$
x \star y:=\sqrt{\frac{1}{2}\left\|\Psi(x)^{-1} E_{1, n+1} \Psi(y)\right\|,}
$$

where $E_{1, n+1}$ is the $(n+1) \times(n+1)$ matrix with one in the $(1, n+1)$-entry and zeros everywhere else. For $x \in U \backslash G$ and $g \in G, x \star x g$ measures the difference between the $U$ components of the Iwasawa decomposition of $x$ and $x g$. More specifically, it measures the $(1, n+1)$ component of $g$. 
For $L \subseteq G$, define

$$
L_{T}:=\{g \in L:\|g\| \leq T\}
$$

and

$$
B_{U}(T):=\left\{u_{\mathbf{t}} \in U:\|\mathbf{t}\| \leq T\right\},
$$

where $\|\mathbf{t}\|$ denotes the max norm of $\mathbf{t} \in \mathbb{R}^{n-1}$. Let $\pi_{U}: G \rightarrow U \backslash G$ denote the natural projection map.

We will be interested in the following quantity:

$$
I(\varphi, T, x):=\int_{P} \mu_{\Psi(x) \Gamma}^{\mathrm{PS}}\left(B_{U}\left(\frac{\sqrt{T}}{x \star \pi_{U}(p)}\right)\right) \varphi\left(\pi_{U}(p)\right) d \nu(p) .
$$

Here, $\varphi$ is a function on $U \backslash G, x \in U \backslash G, T>0, \mu^{\mathrm{PS}}$ denotes the PS measure, fully defined in Sect. 2.2, and $v$ is defined in Sect. 2.3.

For two functions of $T, a(T), b(T)$, we write

$$
a(T) \sim b(T) \Longleftrightarrow \lim _{T \rightarrow \infty} \frac{a(T)}{b(T)}=1 .
$$

We can now state a qualitative version of our ratio theorem:

Theorem 1.2 Let $\Gamma$ be geometrically finite. For any $\varphi \in C_{c}(U \backslash G)$ and every $x \in U \backslash G$ such that $\Psi(x)^{-} \in \Lambda_{r}(\Gamma)$,

$$
\sum_{\gamma \in \Gamma_{T}} \varphi(x \gamma) \sim I(\varphi, T, x) .
$$

The notation $\mathrm{g}^{-}$for $\mathrm{g} \in G$ is defined in Sect. 2.

By the shadow lemma, Proposition 2.1, we obtain the following corollary, which will in turn imply Proposition 1.1:

Corollary 1.3 Assume that $\Gamma$ is convex cocompact. For any $\varphi \in C_{c}(U \backslash G)$ and every $x \in U \backslash G$ such that $\Psi(x)^{-} \in \Lambda(\Gamma)$, as $T \rightarrow \infty$,

$$
\frac{1}{T^{\delta_{\Gamma} / 2}} \sum_{\gamma \in \Gamma_{T}} \varphi(x \gamma) \asymp \int_{P} \frac{\varphi\left(\pi_{U}(p)\right)}{\left(x \star \pi_{U}(p)\right)^{\delta_{\Gamma}}} d \nu(p),
$$

where the implied constant depends on $x$ and $\Gamma$.

Remark 1.4 The proof also works for $\Gamma$ geometrically finite when the geodesic of $\Psi(x) \Gamma$ is bounded. We must then assume that $\Psi(x)^{-} \in \Lambda_{r}(\Gamma)$.

In order to state the quantitative version of Theorem 1.2, we need an additional definition, which gives a precise formulation of the notion that $x \in G / \Gamma$ does not escape to the cusps "too quickly":

Definition 1.5 For $0<\varepsilon<1$ and $s_{0} \geq 1$, we say that $x \in G / \Gamma$ with $x^{-} \in \Lambda(\Gamma)$ is $\left(\varepsilon, s_{0}\right)$-Diophantine if for all $s>s_{0}$,

$$
d\left(\mathcal{C}_{0}, a_{-s} x\right)<(1-\varepsilon) s,
$$

where $\mathcal{C}_{0}$ is a compact set arising from the thick-thin decomposition, and is fully defined in Sect. 2.1. We say that $x \in G / \Gamma$ is $\varepsilon$-Diophantine if it is $\left(\varepsilon, s_{0}\right)$-Diophantine for some $s_{0}$. 
Remark 1.6 A point $x \in G / \Gamma$ is $\varepsilon$-Diophantine for some $\varepsilon>0$ if and only if $x^{-} \in \Lambda_{r}(\Gamma)$, because Definition 1.5 precisely says that $x^{-} \notin \Lambda_{b p}(\Gamma)$, by the construction of the thick-thin decomposition.

When $\Gamma$ is convex cocompact, every $x \in G / \Gamma$ with $x^{-} \in \Lambda(\Gamma)$ is $\varepsilon$-Diophantine for some $\varepsilon$, because all limit points are radial in this case. Observe also that in the lattice case, this condition is always satisfied, because $\Lambda(\Gamma)=\partial\left(\mathbb{H}^{n}\right)$. See [18] for further discussion of this definition.

Definition 1.7 We say that $\Gamma$ satisfies property $\mathbf{A}$ if one of the following holds:

- $\Gamma$ is convex cocompact, or

- $\Gamma$ is geometrically finite, and either

1. $n \leq 4$ and $\mathbb{H}^{n} / \Gamma$ has a cusp of rank $n-1$, or

2. $\delta_{\Gamma}>n-2$.

Remark 1.8 The assumptions on $\Gamma$ in Definition 1.7 are to ensure the effective equidistribution theorem in [18, Theorem 1.4] holds (see Theorem 2.15 for a statement of this theorem in this setting). As discussed in [18], this theorem holds whenever the frame flow satisfies an explicit exponential mixing statement, [18, Assumption 1.1], and this condition is satisfied under the conditions in Definition 1.7. However, Definition 1.7 could be replaced with assuming that the more technical statement [18, Assumption 1.1] is satisfied.

Throughout the paper, the notation

$$
x \ll y
$$

means there exists a constant $c$ such that

$$
x \leq c y .
$$

If a subscript is denoted, e.g. $\ll_{\Gamma}$, this explicitly indicates that this constant depends on $\Gamma$.

Theorem 1.9 Let $\Gamma$ satisfy property $A$. For any $0<\varepsilon<1$, there exist $\ell=\ell(\Gamma) \in \mathbb{N}$ and $\kappa=\kappa(\Gamma, \varepsilon)$ satisfying: for every $\varphi \in C_{c}^{\infty}(U \backslash G)$ and for every $x \in U \backslash G$ such that $\Psi(x) \Gamma$ is $\varepsilon$-Diophantine, and for all $T \gg_{\Gamma, \operatorname{supp} \varphi, x} 1$,

$$
\begin{gathered}
\left|\frac{\sum_{\gamma \in \Gamma_{T}} \varphi(x \gamma)}{\mid \int_{P} \mu_{\Psi(x) \Gamma}^{\mathrm{PS}}\left(B_{U}\left(\frac{\sqrt{T}}{x \star \pi_{U}(p)}\right)\right) \varphi\left(\pi_{U}(p)\right) d \nu(p)}-1\right| \\
\ll_{\Gamma, \operatorname{supp} \varphi, x} T^{-\kappa}\left(1+S_{\ell}(\varphi) v\left(\varphi \circ \pi_{U}\right)^{-1}\right) .
\end{gathered}
$$

The dependencies in this statement are quite explicit. The dependence of $T$ on $x$ in Theorem 1.9 arises from the constant in Lemma 3.2, which is explicitly defined in that proof, and the precise Diophantine nature of $x$, through Theorem 2.15 (i.e. the $\varepsilon$ and $s_{0}$ that appear in Definition 1.5). The implied dependence on $x$ in the conclusion is discussed at the end of Sect. 6.

If the support of the function is small enough, then we can get a more explicit estimate (see Sect. 5). This is used as a main step in the proof of Theorem 1.9.

This paper is organized as follows. In Sect. 2, we present notation used throughout the paper, the definitions and fundamental properties of the measures we are working with, and the equidistribution theorems that will be key in our arguments. In Sect. 3, we explore the duality 
between $\Gamma$ orbits on $U \backslash G$ and of $U$ orbits on $G / \Gamma$, and prove key lemmas that are common to the proofs of both Theorems 1.2 and 1.9. This involves a thickening argument, due to Ledrappier, to reduce the problem to that of equidistribution of $U$ orbits. In Sect. 4, we prove Theorem 1.2, using an equidistribution theorem of Mohammadi and Oh, Theorem 2.14. In Sect. 6, we prove Theorem 1.9, using a quantitative equidistribution theorem, Theorem 2.15. Finally, in Sect. 7, we consider two specific examples, and prove Proposition 1.1.

\section{Notation and preliminary results in $G / \Gamma$}

Let $G=\mathrm{SO}(n, 1)^{\circ}$ and let $\Gamma \subseteq G$ be a Zariski dense discrete subgroup. Let $\pi_{\Gamma}: G \rightarrow G / \Gamma$ be the quotient map.

Let $\Lambda(\Gamma) \subseteq \partial\left(\mathbb{H}^{n}\right)$ denote the limit set of $G / \Gamma$, i.e., the set of all accumulation points of $\Gamma z$ for some $z \in \mathbb{H}^{n} \cup \partial\left(\mathbb{H}^{n}\right)$.

The convex core of $X:=G / \Gamma$ is the image in $X$ of the minimal convex subset of $\mathbb{H}^{n}$ which contains all geodesics connecting any two points in $\Lambda(\Gamma)$.

We say that $\Gamma$ is geometrically finite if a unit neighborhood of the convex core of $\Gamma$ has finite volume.

Fix a reference point $o \in \mathbb{H}^{n}$. Let $K=\operatorname{Stab}_{G}(o)$ and let $d$ denote the left $G$-invariant metric on $G$ which induces the hyperbolic metric on $K \backslash G=\mathbb{H}^{n}$. Fix $w_{o} \in \mathrm{T}^{1}\left(\mathbb{H}^{n}\right)$ and let $M=\operatorname{Stab}_{G}\left(w_{o}\right)$ so that $\mathrm{T}^{1}\left(\mathbb{H}^{n}\right)$ may be identified with $M \backslash G$. For $w \in \mathrm{T}^{1}\left(\mathbb{H}^{n}\right)$,

$$
w^{ \pm} \in \partial \mathbb{H}^{n}
$$

denotes the forward and backward endpoints of the geodesic $w$ determines. For $g \in G$, we define

$$
g^{ \pm}:=w_{o}^{ \pm} g
$$

We say that a limit point $\xi \in \Lambda(\Gamma)$ is radial if there exists a compact subset of $X$ so that some (and hence every) geodesic ray toward $\xi$ has accumulation points in that set. We denote by $\Lambda_{r}(\Gamma)$ the set of all radial limit points.

An element $g \in G$ is called parabolic if the set of fixed points of $g$ in $\partial\left(\mathbb{H}^{n}\right)$ is a singleton. We say that a limit point is parabolic if it is fixed by a parabolic element of $\Gamma$. A parabolic limit point $\xi \in \Lambda(\Gamma)$ is called bounded if the stabilizer $\Gamma_{\xi}$ acts cocompactly on $\Lambda(\Gamma)-\xi$.

We denote by $\Lambda_{r}(\Gamma)$ and $\Lambda_{b p}(\Gamma)$ the set of all radial limit points and the set of all bounded parabolic limit points, respectively. Since $\Gamma$ is geometrically finite (see [2]),

$$
\Lambda(\Gamma)=\Lambda_{r}(\Gamma) \cup \Lambda_{b p}(\Gamma) .
$$

Let $A=\left\{a_{s}: s \in \mathbb{R}\right\}$ be a one parameter diagonalizable subgroup such that $M$ and $A$ commute, and such that the right $a_{s}$ action on $M \backslash G=\mathrm{T}^{1}\left(\mathbb{H}^{n}\right)$ corresponds to unit speed geodesic flow.

We embed $G$ in $\mathrm{SL}_{n+1}(\mathbb{R})$, parametrize $A$ by $A=\left\{a_{s}: s \in \mathbb{R}\right\}$, where

$$
a_{s}=\left(\begin{array}{lll}
e^{s} & & \\
& I & \\
& & e^{-s}
\end{array}\right)
$$


and $I$ denotes the $(n-1) \times(n-1)$ identity matrix, and let

$$
M=\left\{\left(\begin{array}{lll}
1 & & \\
& m & \\
& & 1
\end{array}\right): m \in \operatorname{SO}(n-1)\right\} .
$$

Let $U$ denote the expanding horospherical subgroup

$$
U=\left\{g \in G: a_{-s} g a_{s} \rightarrow e \text { as } s \rightarrow+\infty\right\},
$$

let $\tilde{U}$ be the contracting horospherical subgroup

$$
\tilde{U}=\left\{g \in G: a_{s} g a_{-s} \rightarrow e \text { as } s \rightarrow+\infty\right\},
$$

and let $P=M A \tilde{U}$ be the parabolic subgroup.

The group $U$ is isomorphic to $\mathbb{R}^{n-1}$. We use the parametrization $U=\left\{u_{\mathbf{t}}: \mathbf{t} \in \mathbb{R}^{n-1}\right\}$, where $\mathbf{t}$ is viewed as a row vector, and

$$
u_{\mathbf{t}}=\left(\begin{array}{ccc}
1 & \mathbf{t} & \frac{1}{2}\|\mathbf{t}\|^{2} \\
& I & \mathbf{t}^{T} \\
& & 1
\end{array}\right) .
$$

For more details on these parametrizations and the interactions between these groups, see $[18, \S 2]$.

\subsection{Thick-thin decomposition and the shadow lemma}

There exists a finite set of $\Gamma$-representatives $\xi_{1}, \ldots, \xi_{q} \in \Lambda_{b p}(\Gamma)$. For $i=1, \ldots, q$, fix $g_{i} \in G$ such that $g_{i}^{-}=\xi_{i}$, and for any $R>0$, set

$$
\mathcal{H}_{i}(R):=\bigcup_{s>R} K a_{-s} U g_{i}, \quad \text { and } \quad \mathcal{X}_{i}(R):=\mathcal{H}_{i}(R) \Gamma
$$

(recall, $K=\operatorname{Stab}_{G}(o)$ ). Each $\mathcal{H}_{i}(R)$ is a horoball of depth $R$.

The rank of $\mathcal{H}_{i}(R)$ is the rank of the finitely generated abelian subgroup $\Gamma_{\xi_{i}}=\operatorname{Stab}_{\Gamma}\left(\xi_{i}\right)$. It is known that each rank is strictly smaller than $2 \delta_{\Gamma}$.

Let

$$
\operatorname{supp} m^{\text {BMS }}:=\left\{g \Gamma \in X: g^{ \pm} \in \Lambda(\Gamma)\right\}
$$

Note that the condition $g^{ \pm} \in \Lambda(\Gamma)$ is independent of the choice of representative of $x=g \Gamma$ in the above definition, because $\Lambda(\Gamma)$ is $\Gamma$-invariant. Thus, the notation $x^{ \pm} \in \Lambda(\Gamma)$ is welldefined. For now, supp $m^{\text {BMS }}$ is simply notation, but as we will see, this coincides with the support of the BMS measure, $m^{\mathrm{BMS}}$. We say that a point $x \in X$ is a BMS point if $x \in \operatorname{supp} m^{\text {BMS }}$.

According to [2], there exists $R_{0} \geq 1$ such that $\mathcal{X}_{1}\left(R_{0}\right), \ldots, \mathcal{X}_{q}\left(R_{0}\right)$ are disjoint, and for some compact set $\mathcal{C}_{0} \subset X$,

$$
\operatorname{supp} m^{\mathrm{BMS}} \subseteq \mathcal{C}_{0} \sqcup \mathcal{X}_{1}\left(R_{0}\right) \sqcup \cdots \sqcup \mathcal{X}_{q}\left(R_{0}\right) .
$$




\subsection{Patterson-Sullivan measure}

A family of finite measures $\left\{\mu_{x}: x \in \mathbb{H}^{n}\right\}$ on $\partial\left(\mathbb{H}^{n}\right)$ is called a $\Gamma$-invariant conformal density of dimension $\delta_{\mu}>0$ if for every $x, y \in \mathbb{H}^{n}, \xi \in \partial\left(\mathbb{H}^{n}\right)$ and $\gamma \in \Gamma$,

$$
\gamma_{*} \mu_{x}=\mu_{x \gamma} \text { and } \frac{d \mu_{y}}{d \mu_{x}}(\xi)=e^{-\delta_{\mu} \beta_{\xi}(y, x)},
$$

where $\gamma_{*} \mu_{x}(F)=\mu_{x}(F \gamma)$ for any Borel subset $F$ of $\partial\left(\mathbb{H}^{n}\right)$.

We let $\left\{v_{x}\right\}_{x \in \mathbb{H}^{n}}$ denote the Patterson-Sullivan density on $\partial \mathbb{H}^{n}$, that is, the unique (up to scalar multiplication) conformal density of dimension $\delta_{\Gamma}$.

For each $x \in \mathbb{H}^{n}$, we denote by $m_{x}$ the unique probability measure on $\partial\left(\mathbb{H}^{n}\right)$ which is invariant under the compact subgroup $\operatorname{Stab}_{G}(x)$. Then $\left\{m_{x}: x \in \mathbb{H}^{n}\right\}$ forms a $G$-invariant conformal density of dimension $n-1$, called the Lebesgue density. Fix $o \in \mathbb{H}^{n}$.

For $x, y \in \mathbb{H}^{n}$ and $\xi \in \partial\left(\mathbb{H}^{n}\right)$, the Busemann function is given by

$$
\beta_{\xi}(x, y):=\lim _{t \rightarrow \infty} d\left(x, \xi_{t}\right)-d\left(y, \xi_{t}\right)
$$

where $\xi_{t}$ is a geodesic ray towards $\xi$.

For $g \in G$, we can define measures on $U g$ using the conformal densities defined previously. The Patterson-Sullivan measure (abbreviated as the PS-measure):

$$
d \mu_{U g}^{\mathrm{PS}}\left(u_{\mathbf{t}} g\right):=e^{\delta_{\Gamma} \beta_{\left(u_{\mathbf{t}} g\right)^{+}}\left(o, u_{\mathbf{t}} g(o)\right)} d v_{o}\left(\left(u_{\mathbf{t}} g\right)^{+}\right),
$$

and the Lebesgue measure

$$
d \mu_{U g}^{\mathrm{Leb}}\left(u_{\mathbf{t}} g\right):=e^{(n-1) \beta_{\left(u_{\mathbf{t}}\right)^{+}}\left(o, u_{\mathbf{t}} g(o)\right)} d m_{o}\left(\left(u_{\mathbf{t}} g\right)^{+}\right) .
$$

Note that for any $g \in G$, a point $h \in U g$ satisfies $h \in \operatorname{supp} \mu_{U g}^{\mathrm{PS}}$ if and only if $h^{+} \in \Lambda(\Gamma)$. Therefore, we refer to the points $x \in X$ which satisfy $x^{+} \in \Lambda(\Gamma)$ as PS points.

The conformal properties of $m_{x}$ and $v_{x}$ imply that this definition is independent of the choice of $o \in \mathbb{H}^{n}$.

We often view $\mu_{U g}^{\mathrm{PS}}$ as a measure on $U$ via

$$
d \mu_{g}^{\mathrm{PS}}(\mathbf{t}):=d \mu_{U g}^{\mathrm{PS}}\left(u_{\mathbf{t}} g\right)
$$

The measure

$$
d \mu_{U g}^{\mathrm{Leb}}\left(u_{\mathbf{t}} g\right)=d \mu_{U}^{\mathrm{Leb}}\left(u_{\mathbf{t}}\right)=d \mathbf{t}
$$

is independent of the orbit $U g$ and is simply the Lebesgue measure on $U \equiv \mathbb{R}^{n-1}$ up to a scalar multiple.

If $x \in X$ is such that $x^{-} \in \Lambda_{r}(\Gamma)$, then

$$
u \mapsto u x
$$

is injective, and we can define the PS measure on $U x \subseteq X$, denoted $\mu_{x}^{\mathrm{PS}}$, simply by pushforward of $\mu_{g}^{\mathrm{PS}}$, where $x=g \Gamma$. In general, defining $\mu_{x}^{\mathrm{PS}}$ requires more care, see e.g. [14, §2.3] for more details. As before, we can view $\mu_{x}^{\mathrm{PS}}$ as a measure on $U$ via

$$
d \mu_{x}^{\mathrm{PS}}(\mathbf{t})=d \mu_{x}^{\mathrm{PS}}\left(u_{\mathbf{t}} x\right) .
$$

Recall that for $T>0$,

$$
B_{U}(T):=\left\{u_{\mathbf{t}}:\|\mathbf{t}\| \leq T\right\},
$$


where $\|\mathbf{t}\|$ is the max norm of $\mathbf{t}$ as measured in $\mathbb{R}^{n-1}$.

We will need the following version of Sullivan's shadow lemma:

Proposition 2.1 ([12, Proposition 5.1, Remark 5.2])

There exists a constant $\lambda=\lambda(\Gamma) \geq 1$ such that for all $x \in \operatorname{supp} m^{\mathrm{BMS}}$ and all $T>0$, we have

$$
\begin{aligned}
\left.\lambda^{-1} T^{\delta_{\Gamma}} e^{\left(k(x, T)-\delta_{\Gamma}\right) d\left(\mathcal{C}_{0}, a_{-} \log T\right.} x\right) & \leq \mu_{x}^{\mathrm{PS}}\left(B_{U}(T)\right) \\
& \leq \lambda T^{\delta_{\Gamma}} e^{\left(k(x, T)-\delta_{\Gamma}\right) d\left(\mathcal{C}_{0}, a_{-\log T} x\right)},
\end{aligned}
$$

where $k(x, T)$ denotes the rank of the cusp containing $a_{-\log T} x$ (and is zero if $a_{-\log T} x \in \mathcal{C}_{0}$ ).

Remark 2.2 In [12], the shadow lemma is proven using the distance measured in $\mathbb{H}^{n} / \Gamma$. However, because $\mathcal{C}_{0}$ is $K$-invariant and $\mathbb{H}^{n}=K \backslash G$, we obtain the form above.

Remark 2.3 When $\Gamma$ is convex cocompact, $\mathcal{C}_{0}=\operatorname{supp} m^{\mathrm{BMS}}$, and the shadow lemma simplifies to

$$
\lambda^{-1} T^{\delta_{\Gamma}} \leq \mu_{x}^{\mathrm{PS}}\left(B_{U}(T)\right) \leq \lambda T^{\delta_{\Gamma}} .
$$

We will need the following, which says that the PS measure is doubling.

Lemma 2.4 ([18, Corollary 9.9]) There exist constants $\sigma_{1}=\sigma_{1}(\Gamma) \geq \delta_{\Gamma}, \sigma_{2}=\sigma_{2}(\Gamma)>0$ such that for every $c>0$, every $x \in \operatorname{supp} m^{\mathrm{BMS}}$ and every $T>0$,

$$
\mu_{x}^{\mathrm{PS}}\left(B_{U}(c T)\right) \ll_{\Gamma} \max \left\{c^{\sigma_{1}}, c^{\sigma_{2}}\right\} \mu_{x}^{\mathrm{PS}}\left(B_{U}(T)\right) .
$$

We will also require control of the PS measure of slightly larger balls, specifically as will be established below in Lemma 2.9. This will be a result of the friendliness of the PS density when $\Gamma$ is geometrically finite, established in [3]. More specifically, we will show that the measure of the boundary of certain balls can be controlled.

Let $d$ be a left-invariant Riemannian metric on $G / \Gamma$ that projects to the hyperbolic distance on $\mathbb{H}^{n}$.

Denote by $d_{E}$ the Euclidean metric on $\mathbb{R}^{n-1}$. For a subset $S \subseteq \mathbb{R}^{n-1}$ and $\xi>0$, let

$$
\mathcal{N}(S, \xi)=\left\{x \in \mathbb{R}^{n-1}: d_{E}(x, S) \leq \xi\right\} .
$$

For $v \in \mathbb{R}^{n-1}$ and $r>0$, let

$$
B(v, r)=\left\{u \in \mathbb{R}^{n-1}: d_{E}(u, v) \leq r\right\}
$$

be the Euclidean ball of radius $r$ around $v$.

We say that a hyperplane $L$ is on the boundary of a closed ball $B$ if

$$
\emptyset \neq L \cap B \subseteq \partial(B) .
$$

Below, we obtain estimates for the PS measure of small neighbourhoods of hyperplanes on the boundary of a ball centered at a BMS point. Though not written here, estimates also hold when the center of the ball is a PS point but not a BMS point, as long as the ball is sufficiently small. In this case, one may use arguments similar to those in the appendix of [18].

We caution the reader that the estimates below hold only for hyperplanes on the boundary of such a ball; to obtain such estimates for general hyperplanes, absolute friendliness of the PS density is necessary. By [3, Theorem 1.9], this is satisfied if and only if all cusps of $\mathbb{H}^{n} / \Gamma$ are of maximal rank $n-1$ (note that this is vacuously satisfied if $\Gamma$ is convex cocompact). In this case, one may use [18, Corollary 9.14] when $\Gamma$ is geometrically finite or $[17$, Theorem $2]$ if $\Gamma$ is convex cocompact. 
Lemma 2.5 There exists a constant $\alpha=\alpha(\Gamma)>0$ satisfying the following: for all $\lambda \in \Lambda(\Gamma)$, $\xi>0,0<\eta \leq 1$, and every hyperplane $L$ that is on the boundary of $B(\lambda, \eta)$, we have that

$$
v_{o}(\mathcal{N}(L, \xi) \cap B(\lambda, \eta)) \ll_{\Gamma}\left(\frac{\xi}{\eta}\right)^{\alpha} v_{o}(B(\lambda, \eta)) .
$$

Proof By [3, Theorem 1.9], $v_{o}$ is friendly when $\Gamma$ is geometrically finite. In particular, this means that there exists $\alpha=\alpha(\Gamma)>0$ such that for all $\lambda \in \Lambda(\Gamma), \xi>0,0<\eta \leq 1$, and every affine hyperplane $L \subseteq \partial\left(\mathbb{H}^{n}\right)$,

$$
v_{o}\left(\mathcal{N}\left(L, \xi\left\|d_{L}\right\|_{v_{o}, B(\lambda, \eta)}\right) \cap B(\lambda, \eta)\right) \ll_{\Gamma} \xi^{\alpha} v_{o}(B(\lambda, \eta)),
$$

where

$$
\left\|d_{L}\right\|_{v_{o}, B(\lambda, \eta)}:=\sup \{d(\mathbf{y}, L): \mathbf{y} \in B(\lambda, \eta) \cap \Lambda(\Gamma)\} .
$$

Since $\lambda \in \Lambda(\Gamma)$, for any $L$ that is on the boundary of $B(\lambda, \eta)$, we have that

$$
\left\|d_{L}\right\|_{v_{o}, B(\lambda, \eta)} \geq \eta / 2 .
$$

Thus, for any $L$ that is on the boundary of $B(\lambda, \eta)$, we have

$$
v_{o}(\mathcal{N}(\xi \eta / 2) \cap B(\lambda, \eta)) \ll \Gamma \xi^{\alpha} v_{o}(B(\lambda, \eta)) .
$$

Replacing $\xi$ with $2 \xi \eta^{-1}$ then implies that for every such $L$,

$$
v_{o}(\mathcal{N}(L, \xi) \cap B(\lambda, \eta)) \ll_{\Gamma}\left(\frac{\xi}{\eta}\right)^{\alpha} v_{o}(B(\lambda, \eta)),
$$

as desired.

By flowing with $a_{-s}$ for $s>0$, we obtain similar estimates for large balls centered at BMS points:

Corollary 2.6 Let $\alpha=\alpha(\Gamma)>0$ be as in Lemma 2.5. For every $x \in \operatorname{supp} m^{\text {BMS }}$ such that $x^{-} \in \Lambda_{r}(\Gamma)$, every $\eta, \xi>0$, and every hyperplane $L$ in the boundary of $B_{U}(\eta) x$, we have

$$
\mu_{x}^{\mathrm{PS}}\left(\mathcal{N}_{U}(L, \xi) \cap B_{U}(\eta)\right) \ll_{\Gamma}\left(\frac{\xi}{\eta}\right)^{\alpha} \mu_{x}^{\mathrm{PS}}\left(B_{U}(\eta)\right) .
$$

Proof We will first prove that there exists a constant $c=c(\Gamma)>0$ so that for all $x \in$ supp $m^{\text {BMS }} \cap \mathcal{C}_{0}$ with $x^{-} \in \Lambda_{r}(\Gamma), \xi>0, \eta$ satisfying

$$
0<\eta \leq c^{-1}
$$

and every hyperplane $L$ in the boundary of $B_{U}(\eta) x$, the inequality in the statement is satisfied.

Since $\mathcal{C}_{0}$ is compact, there exists $c=c(\Gamma)>1$ such that for any $x \in \mathcal{C}_{0}$ we can find $g \in G$ such that $x=g \Gamma$ and $d(o, g(o))<c$. Then, for any $u_{\mathbf{t}} \in B_{U}\left(c^{-1}\right)$, we have

$$
\begin{aligned}
\left|\beta_{\left(u_{\mathbf{t}} g\right)^{+}}\left(o, u_{\mathbf{t}} g(o)\right)\right| & \leq d\left(u_{\mathbf{t}}^{-1}(o), g(o)\right) \\
& \leq d\left(u_{-\mathbf{t}}(o), o\right)+d(o, g(o)) \\
& \leq 2 c .
\end{aligned}
$$

Then, by the definition of the PS measure, we have

$$
\mu_{x}^{\mathrm{PS}}\left(\mathcal{N}_{U}(L, \xi) \cap B_{U}(\eta)\right)=\mu_{g}^{\mathrm{PS}}\left(\mathcal{N}_{U}(L, \xi) \cap B_{U}(\eta)\right)
$$




$$
\begin{aligned}
& =\int_{\mathbf{t} \in \mathcal{N}_{U}(L, \xi) \cap B_{U}(\eta)} e^{\delta_{\Gamma} \beta_{\left(u_{\mathbf{t} g}\right)^{+}}\left(o, u_{\mathbf{t}} g(o)\right)} d v_{o}\left(\left(u_{\mathbf{t}} g\right)^{+}\right) \\
& \ll \Gamma v_{o}\left(\operatorname{Pr}_{g^{-}}\left(\mathcal{N}(L, \xi) \cap B_{U}(\eta)\right)\right),
\end{aligned}
$$

where $\operatorname{Pr}_{g^{-}}: U g \rightarrow \partial\left(\mathbb{H}^{n}\right) \backslash\left\{g^{-}\right\}$is the visual map $w \mapsto w^{+}$. Using [18, Corollary 9.5], we may assume that $c$ satisfies

$$
v_{o}\left(\operatorname{Pr}_{g^{-}}\left(\mathcal{N}(L, \xi) \cap B_{U}(\eta)\right)\right) \ll \Gamma v_{o}\left(\mathcal{N}\left(L^{\prime}, c^{2} \xi\right) \cap B\left(g^{+}, c^{2} \eta\right)\right),
$$

where $L^{\prime}$ is a hyperplane in the boundary obtained from the projection of $L$ under the visual map $\operatorname{Pr}_{g^{-}}$. Thus, for any $x \in \mathcal{C}_{0}$ and $u_{\mathbf{t}} \in B_{U}(c)$, we arrive at

$$
\mu_{x}^{\mathrm{PS}}\left(\mathcal{N}_{U}(L, \xi) \cap B_{U}(\eta)\right) \ll_{\Gamma} v_{o}\left(\mathcal{N}\left(L^{\prime}, c^{2} \xi\right) \cap B\left(g^{+}, c^{2} \eta\right)\right)
$$

and in a similar way, one may also deduce

$$
\mu_{x}^{\mathrm{PS}}\left(B_{U}(\eta)\right) \gg_{\Gamma} v_{o}\left(B\left(g^{+}, c^{-2} \eta\right)\right),
$$

Now, we may conclude

$$
\begin{aligned}
\mu_{x}^{\mathrm{PS}}\left(\mathcal{N}_{U}(L, \xi) \cap B_{U}(\eta)\right) & \ll \Gamma v_{o}\left(\mathcal{N}\left(L^{\prime}, c^{2} \xi\right) \cap B\left(g^{+}, c^{2} \eta\right)\right) \\
& \ll \Gamma\left(\frac{\xi}{\eta}\right)^{\alpha} v_{o}\left(B\left(g^{+}, c^{2} \eta\right)\right) \\
& \ll \Gamma\left(\frac{\xi}{\eta}\right)^{\alpha} v_{o}\left(B\left(g^{+}, c^{-2} \eta\right)\right) \\
& \ll \Gamma\left(\frac{\xi}{\eta}\right)^{\alpha} \mu_{x}^{\mathrm{PS}}\left(B_{U}(\eta)\right)
\end{aligned}
$$

Now, let $x \in \operatorname{supp} m^{\text {BMS }}$ with $x^{-} \in \Lambda_{r}(\Gamma)$ and let $\eta>0$. Since $a_{-s} x$ has accumulation points in $\mathcal{C}_{0}$, there exists $s>0$ so that $e^{-s} \eta<c^{-1}$ and $a_{-s} x \in \mathcal{C}_{0}$. By the first step of the proof, we then have that

$$
\begin{aligned}
\frac{\mu_{x}^{\mathrm{PS}}\left(\mathcal{N}_{U}(L, \xi) \cap B_{U}(\eta)\right)}{\mu_{x}^{\mathrm{PS}}\left(B_{U}(\eta)\right)} & =\frac{\mu_{a_{-s} x}^{\mathrm{PS}}\left(\mathcal{N}_{U}\left(L, e^{-s} \xi\right) \cap B_{U}\left(e^{-s} \eta\right)\right)}{\mu_{a_{-s} x}^{\mathrm{PS}}\left(B_{U}\left(e^{-s} \eta\right)\right)} \\
& \ll \Gamma\left(\frac{e^{-s} \xi}{e^{-s} \eta}\right)^{\alpha}=\left(\frac{\xi}{\eta}\right)^{\alpha}
\end{aligned}
$$

Proposition 2.7 Let $\alpha=\alpha(\Gamma)>0$ be as in Corollary 2.6. Then for all $x \in \operatorname{supp} m^{\mathrm{BMS}}$ such that $x^{-} \in \Lambda_{r}(\Gamma), T>0$, and $0<\varepsilon \leq 1$, we have that

$$
\mu_{x}^{\mathrm{PS}}\left(B_{U}((1+2 \varepsilon) T)\right)-\mu_{x}^{\mathrm{PS}}\left(B_{U}(T)\right) \ll_{\Gamma} \varepsilon^{\alpha} \mu_{x}^{\mathrm{PS}}\left(B_{U}(T)\right) .
$$

Proof By the geometry of $\left(B_{U}((1+2 \varepsilon) T)-B_{U}(T)\right) x$, there exists a constant $m$ depending only on $n$ and hyperplanes $L_{1}, \ldots, L_{m}$ in the boundary of $B_{U}((1+2 \varepsilon) T) x$ so that

$$
\left(B_{U}((1+2 \varepsilon) T)-B_{U}(T)\right) x \subseteq \bigcup_{i=1}^{m} \mathcal{N}_{U}\left(L_{i}, 2 \varepsilon T\right) \cap B_{U}((1+2 \varepsilon) T) x .
$$

Then by Corollary 2.6, we have that

$$
\mu_{x}^{\mathrm{PS}}\left(B_{U}((1+2 \varepsilon) T)\right)-\mu_{x}^{\mathrm{PS}}\left(B_{U}(T)\right)
$$




$$
\begin{aligned}
& \leq \sum_{i=1}^{m} \mu_{x}^{\mathrm{PS}}\left(\mathcal{N}_{U}\left(L_{i}, 2 \varepsilon T\right) \cap B_{U}((1+2 \varepsilon) T)\right) \\
& \ll_{\Gamma}\left(\frac{2 \varepsilon T}{(1+2 \varepsilon) T}\right)^{\alpha} \mu_{x}^{\mathrm{PS}}\left(B_{U}((1+2 \varepsilon) T)\right) \\
& \ll_{\Gamma} \varepsilon^{\alpha} \mu_{x}^{\mathrm{PS}}\left(B_{U}(T)\right)
\end{aligned}
$$

by Lemma 2.4

Note that the assumption $\varepsilon \leq 1$ is for convenience in the last step only; one may still use Lemma 2.4 if $\varepsilon$ is not bounded, but the exponent on $\varepsilon$ must change.

We can obtain estimates for all $\left(\varepsilon, s_{0}\right)$-Diophantine points for balls that are sufficiently large (in a way that is uniform and linear in $s_{0}$ ). In fact, for any compact set $\Omega \subseteq G / \Gamma$, there exists a $T_{0}=T_{0}(\Omega)$ satisfying the statement below for all $x \in \Omega$ with $x^{-} \in \Lambda_{r}(\Gamma)$, see e.g. $[14$, Lemma 3.3]. Thus, the statement below could take many forms and this is not as strong as possible; we simply write it in a way that is useful for our setting.

Corollary 2.8 Let $\alpha=\alpha(\Gamma)>0$ be as in Proposition 2.7, let $0<\varepsilon \leq 1$ and let $s_{0} \geq 1$. There exists $T_{0}=T_{0}\left(\Gamma, s_{0}\right)>0$ so that for every $\left(\varepsilon, s_{0}\right)$-Diophantine point $x \in G / \Gamma$, all $T \geq 2 T_{0}+1$, and all $\xi>0$,

$$
\mu_{x}^{\mathrm{PS}}\left(B_{U}((1+2 \xi) T)\right)-\mu_{x}^{\mathrm{PS}}\left(B_{U}(T)\right) \ll_{\Gamma}\left(\xi+\frac{T_{0}}{T-T_{0}}\right)^{\alpha} \mu_{x}^{\mathrm{PS}}\left(B_{U}(T)\right) .
$$

In particular, if $x^{-} \in \Lambda_{r}(\Gamma)$, there exists $T_{0}=T_{0}(x)>0$ so that for all $T \geq 2 T_{0}+1$ and all $\xi>0,(10)$ holds.

Proof By [18, Lemma 3.8], there exists $T_{0}=T_{0}\left(\Gamma, s_{0}\right)>0$ (in fact, it is linear in $s_{0}$ ) so that for every $\left(\varepsilon, s_{0}\right)$-Diophantine point $x$, there exists

$$
y \in B_{U}\left(T_{0}\right) x \cap \operatorname{supp} m^{\text {BMS }} .
$$

For $T \geq T_{0}$, we have

$$
B_{U}\left(T-T_{0}\right) y \subseteq B_{U}(T) x \subseteq B_{U}\left(T+T_{0}\right) y .
$$

In particular,

$$
B_{U}((1+2 \xi) T) x \subseteq B_{U}\left((1+2 \xi)\left(T+T_{0}\right)\right) y
$$

and

$$
B_{U}\left(T-T_{0}\right) y \subseteq B_{U}(T) x .
$$

Since all Diophantine points are radial, by assuming that $T \geq 2 T_{0}+1$, we may use Proposition 2.7 below:

$$
\begin{aligned}
& \mu_{x}^{\mathrm{PS}}\left(B_{U}(1+2 \xi) T\right)-\mu_{x}^{\mathrm{PS}}\left(B_{U}(T)\right) \\
& \quad \leq \mu_{y}^{\mathrm{PS}}\left(B_{U}(1+2 \xi)\left(T+T_{0}\right)\right)-\mu_{y}^{\mathrm{PS}}\left(B_{U}\left(T-T_{0}\right)\right) \\
& \quad \leq \mu_{y}^{\mathrm{PS}}\left(B_{U}\left((1+2 \xi)\left(1+\frac{2 T_{0}}{T-T_{0}}\right)\left(T-T_{0}\right)\right)\right)-\mu_{y}^{\mathrm{PS}}\left(B_{U}\left(T-T_{0}\right)\right) \\
& \quad \ll \Gamma\left(\xi+\frac{T_{0}}{T-T_{0}}+\frac{\xi T_{0}}{T-T_{0}}\right)^{\alpha} \mu_{y}^{\mathrm{PS}}\left(B_{U}\left(T-T_{0}\right)\right) \text { by Proposition } 2.7 \\
& \quad \ll \Gamma\left(\xi+\frac{(1+\xi) T_{0}}{T-T_{0}}\right)^{\alpha} \mu_{x}^{\mathrm{PS}}\left(B_{U}(T)\right)
\end{aligned}
$$


Since $T \geq 2 T_{0}+1$,

$$
\frac{\xi T_{0}}{T-T_{0}} \leq \xi
$$

and it can be absorbed into the $\xi$ term, completing the proof.

We may now state the form in which we will need this control. The implied constant below depends on $x$ through the initial time in Corollary 2.8, so it can be made uniform over a compact set or over all points with the same Diophantine properties. However, this level of detail is not necessary for our results.

Lemma 2.9 Let $\alpha=\alpha(\Gamma)>0$ be as in Corollary 2.8. For every $x \in G / \Gamma$ with $x^{-} \in \Lambda_{r}(\Gamma)$, $c>0,0<\eta \leq 1$, and $0<r_{+}<\ell<r_{-}$satisfying

$$
\frac{r_{+}}{r_{-}}<1+\eta
$$

there exists $T_{0}=T_{0}\left(x, r_{+}, r_{-}\right)>0$ such that for any $T>T_{0}$,

$$
\begin{aligned}
& \left|\mu_{x}^{\mathrm{PS}}\left(B_{U}\left(\frac{\sqrt{T} \pm c}{r_{ \pm}} \pm \eta\right)\right)-\mu_{x}^{\mathrm{PS}}\left(B_{U}\left(\frac{\sqrt{T}}{\ell}\right)\right)\right| \\
& \ll_{\Gamma, x}\left(\eta+\frac{c+1}{\sqrt{T}}\right)^{\alpha} \mu_{x}^{\mathrm{PS}}\left(B_{U}\left(\frac{\sqrt{T}}{\ell}\right)\right)
\end{aligned}
$$

Proof First, observe that by Corollary 2.8, there exists $T_{1}=T_{1}(x)$ so that for all $T \geq 2 T_{1}+1$ and all $\xi>0$,

$$
\frac{\mu_{x}^{\mathrm{PS}}\left(B_{U}(T+\xi)\right)-\mu_{x}^{\mathrm{PS}}\left(B_{U}(T)\right)}{\mu_{x}^{\mathrm{PS}}\left(B_{U}(T)\right)} \ll_{\Gamma}\left(\xi+\frac{T_{1}}{T-T_{1}}\right)^{\alpha} \ll_{\Gamma}\left(\xi+\frac{T_{1}}{T}\right)^{\alpha} .
$$

This follows immediately from the fact that

$$
\mu_{x}^{\mathrm{PS}}\left(B_{U}(T+\xi)\right) \leq \mu_{x}^{\mathrm{PS}}\left(B_{U}(1+2 \xi) T\right) .
$$

Thus, if we assume that $T$ is sufficiently large so that $\sqrt{T} / \ell \geq 2 T_{1}+1$ (and note that this condition can be taken to rely on $r_{-}$rather than on $\ell$ specifically), and note that by the assumption,

$$
1 \leq \frac{\ell}{r_{+}} \leq 1+\eta
$$

we see from (12) that

$$
\begin{aligned}
& \mu_{x}^{\mathrm{PS}}\left(B_{U}\left(\frac{\sqrt{T}+c}{r_{+}}+\eta\right)\right)-\mu_{x}^{\mathrm{PS}}\left(B_{U}\left(\frac{\sqrt{T}}{\ell}\right)\right) \\
& \ll \Gamma\left(\frac{r_{+}^{-1}(\sqrt{T}+c)+\eta-\ell^{-1} \sqrt{T}}{\ell^{-1} \sqrt{T}}+\frac{T_{1}}{\ell^{-1} \sqrt{T}}\right)^{\alpha} \mu_{x}^{\mathrm{PS}}\left(B_{U}\left(\frac{\sqrt{T}}{\ell}\right)\right) \\
& \ll \Gamma\left(\frac{\ell r_{+}^{-1}(\sqrt{T}+c)+\ell \eta-\sqrt{T}+T_{1}}{\sqrt{T}}\right)^{\alpha} \mu_{x}^{\mathrm{PS}}\left(B_{U}\left(\frac{\sqrt{T}}{\ell}\right)\right)
\end{aligned}
$$




$$
\begin{aligned}
& \ll_{\Gamma}\left(\frac{(1+\eta)(\sqrt{T}+c)+\ell \eta-\sqrt{T}+T_{1}}{\sqrt{T}}\right)^{\alpha} \mu_{x}^{\mathrm{PS}}\left(B_{U}\left(\frac{\sqrt{T}}{\ell}\right)\right) \\
& \ll_{\Gamma}\left(\eta+\frac{c+\eta \ell+T_{1}}{\sqrt{T}}\right)^{\alpha} \mu_{x}^{\mathrm{PS}}\left(B_{U}\left(\frac{\sqrt{T}}{\ell}\right)\right) \\
& \ll_{\Gamma, x}\left(\eta+\frac{c+\eta \ell+1}{\sqrt{T}}\right)^{\alpha} \mu_{x}^{\mathrm{PS}}\left(B_{U}\left(\frac{\sqrt{T}}{\ell}\right)\right)
\end{aligned}
$$

Note that the implied constant depends on $x$ because we have absorbed the constant $T_{1}$. Now, choose $T_{0} \geq T_{1}$ so that $T \geq T_{0}$ implies $\frac{\ell}{\sqrt{T}}<1$ (a condition which depends on $x$ and $r_{-}$in this case), which implies the claim because we may then absorb this term into the $\eta$ term.

The second case can be shown in a similar way, with the choice of $T_{0}$ depending on $x$ and $r_{+}$there.

\subsection{Burger-Roblin and Bowen-Margulis-Sullivan measures}

Let $\pi: \mathrm{T}^{1}\left(\mathbb{H}^{n}\right) \rightarrow \mathbb{H}^{n}$ be the natural projection. Recalling the fixed reference point $o \in \mathbb{H}^{n}$ as before, the map

$$
w \mapsto\left(w^{+}, w^{-}, s:=\beta_{w^{-}}(o, \pi(w))\right)
$$

is a homeomorphism between $\mathrm{T}^{1}\left(\mathbb{H}^{n}\right)$ and

$$
\left(\partial\left(\mathbb{H}^{n}\right) \times \partial\left(\mathbb{H}^{n}\right)-\left\{(\xi, \xi): \xi \in \partial\left(\mathbb{H}^{n}\right)\right\}\right) \times \mathbb{R} .
$$

This homeomorphism allows us to define the Bowen-Margulis-Sullivan (BMS) and the Burger-Roblin (BR) measure on $\mathrm{T}^{1}\left(\mathbb{H}^{n}\right)$, denoted by $\tilde{m}^{\mathrm{BMS}}$ and $\tilde{m}^{\mathrm{BR}}$, respectively:

$$
\begin{aligned}
d \tilde{m}^{\mathrm{BMS}}(w) & :=e^{\delta_{\Gamma} \beta_{w^{+}}(o, \pi(w))} e^{\delta_{\Gamma} \beta_{w^{-}}(o, \pi(w))} d v_{o}\left(w^{+}\right) d v_{o}\left(w^{-}\right) d s, \\
d \tilde{m}^{\mathrm{BR}}(w) & :=e^{(n-1) \beta_{w^{+}}(o, \pi(w))} e^{\delta_{\Gamma} \beta_{w^{-}}(o, \pi(w))} d m_{o}\left(w^{+}\right) d v_{o}\left(w^{-}\right) d s .
\end{aligned}
$$

The conformal properties of $\left\{v_{x}\right\}$ and $\left\{m_{x}\right\}$ imply that these definitions are independent of the choice of $o \in \mathbb{H}^{n}$. Using the identification of $\mathrm{T}^{1}\left(\mathbb{H}^{n}\right)$ with $M \backslash G$, we lift the above measures to $G$ so that they are all invariant under $M$ from the left. By abuse of notation, we use the same notation $\left(\tilde{m}^{\mathrm{BMS}}\right.$ and $\left.\tilde{m}^{\mathrm{BR}}\right)$. These measures are left $\Gamma$-invariant, and hence induce locally finite Borel measures on $X$, which are the Bowen-Margulis-Sullivan measure $m^{\mathrm{BMS}}$ and the Burger-Roblin measure $m^{\mathrm{BR}}$, respectively.

Note that

$$
\operatorname{supp}\left(m^{\mathrm{BR}}\right)=\left\{x \in X: x^{-} \in \Lambda(\Gamma)\right\},
$$

and the support of the BMS measure indeed satisfies (4).

Recall the definition of $\tilde{U}$, and $P=M A \tilde{U}$ from the begining of Sect. 2. $P$ is the stabilizer of $w_{o}^{+}$in $G$. Hence, one can define a measure $v$ on $P g$ for $g \in G$, which will give us a product structure for $\tilde{m}^{\text {BMS }}$ and $\tilde{m}^{\text {BR }}$ that will be useful in our approach. For any $g \in G$, define

$$
d v(p g):=e^{\delta_{\Gamma} \beta_{(p g)^{-}}(o, p g(o))} d v_{o}\left(w_{o}^{-} p g\right) d m d s,
$$

on $P g$, where $s=\beta_{(p g)^{-}}(o, p g(o)), p=m a v \in M A \tilde{U}$ and $d m$ is the probability Haar measure on $M$. 
Then for any $\psi \in C_{c}(G)$ and $g \in G$, we have

$$
\tilde{m}^{\mathrm{BR}}(\psi)=\int_{P g} \int_{U} \psi\left(u_{\mathbf{t}} p g\right) d \mathbf{t} d v(p g)
$$

and

$$
\tilde{m}^{\mathrm{BMS}}(\psi)=\int_{P g} \int_{U} \psi\left(u_{\mathbf{t}} p g\right) d \mu_{p g}^{\mathrm{PS}}(\mathbf{t}) d \nu(p g) .
$$

\subsection{Sobolev norms}

In the next section we formulate the equidistribution and effective equidistribution results which we will use in the proof of the main theorems. In order to formulate them, we first need to define Sobolev norms. Our proofs will require constructing smooth indicator functions and partitions of unity with controlled Sobolev norms. This section also includes lemmas constructing such partitions.

For $\ell \in \mathbb{N}, 1 \leq p \leq \infty$, and $\psi \in C^{\infty}(X) \cap L^{p}(X)$ we consider the following Sobolev norm

$$
S_{p, \ell}(\psi)=\sum\|U \psi\|_{p}
$$

where the sum is taken over all monomials $U$ in a fixed basis of $\mathfrak{g}=\operatorname{Lie}(G)$ of order at most $\ell$, and $\|\cdot\|_{p}$ denotes the $L^{p}(X)$-norm. Since we will be using $S_{2, \ell}$ most often, we set

$$
S_{\ell}=S_{2, \ell}
$$

Lemma 2.10 ([9, Lemma 2.4.7])

(1) Let $X, Y$ be Riemannian manifolds, and let $\varphi \in C_{c}^{\infty}(X), \psi \in C_{c}^{\infty}(Y)$. Consider $\varphi \cdot \psi$ as a function on $X \times Y$. Then

$$
S_{\ell}(\varphi \cdot \psi) \leq c(X, Y) S_{\ell}(\varphi) S_{\ell}(\psi),
$$

where $c(X, Y)$ is a constant depending only on $X$ and $Y$ (independent of $\varphi, \psi$ ).

(2) Let $X$ be a Riemannian manifold of dimension $N$ and let $x \in X$. Then for any $0<r<1$, there exists a non-negative function $f \in C_{c}^{\infty}(X)$ such that $\operatorname{supp}(f)$ is contained in the ball of radius $r$ centered at $x, \int_{X} f=1$, and

$$
S_{\ell}(f) \leq c(X, x) r^{-\ell+N / 2},
$$

where $c(X, x)$ is a constant depending only on $X$ and $x$, not $r$.

The following lemma is an immediate consequence of the product rule.

Lemma 2.11 Let $X$ be a Riemannian manifold and let $\varphi, \psi \in C_{c}^{\infty}(X)$. For any $\ell \in \mathbb{N}$,

$$
S_{\ell}(\varphi \cdot \psi) \ll_{\ell} S_{\ell}(\varphi) S_{\ell}(\psi)
$$

Lemma 2.12 For any $\ell^{\prime}$ there exists $\ell>\ell^{\prime}$ which satisfies the following. Let $X, Y$ be Riemannian manifolds, $\varphi \in C_{c}^{\infty}(X)$, and $\psi: Y \rightarrow X$ be a smooth function. Then

$$
S_{\ell^{\prime}}(\varphi \circ \psi) \ll_{\ell^{\prime}, \psi} S_{\ell}(\varphi) .
$$


Proof By the chain rule, for any $1 \leq k \leq \ell^{\prime}$,

$$
\begin{aligned}
\left\|(\varphi \circ \psi)^{(k)}\right\|_{2} & \ll \psi, k \sum_{i=0}^{k}\left\|\varphi^{(i)} \circ \psi\right\|_{2} \\
& \ll \psi, k \sum_{i=0}^{k}\left\|\varphi^{(i)}\right\|_{\infty} m^{\text {Haar }}(\operatorname{supp} \varphi) \\
& \ll \psi, k \\
& \ll_{\infty, \ell^{\prime}}(\varphi) m^{\text {Haar }}(\operatorname{supp} \varphi)
\end{aligned}
$$

where in the last line, we have used [1] to choose $\ell>\ell^{\prime}$ satisfying

$$
S_{\infty, \ell^{\prime}}(f) m^{\text {Haar }}(\operatorname{supp} f) \ll S_{\ell}(f)
$$

for any $f$, where the implied constant is global.

Lemma 2.13 Let $H$ be a Riemannian manifold of dimension $N, 0<r<1, \ell \in \mathbb{N}$, and $E$ a bounded subset of $H$. Then, there exists a partition of unity $\sigma_{1}, \ldots, \sigma_{k}$ of $E$ in $H_{r}(E)=$ $\left\{g \in G: d_{H}(g, E) \leq r\right\}$ where $d_{H}$ denotes the Riemannian metric on H, i.e.

$$
\sum_{i=1}^{k} \sigma_{i}(x)= \begin{cases}0 & \text { if } x \notin H_{r}(E) \\ 1 & \text { if } x \in E,\end{cases}
$$

such that for some $u_{1}, \ldots, u_{k} \in E$ and all $1 \leq i \leq k$

$$
\sigma_{i} \in C_{c}^{\infty}\left(H_{r}\left(u_{i}\right)\right), \quad S_{\ell}\left(\sigma_{i}\right) \ll_{N} r^{-\ell+N / 2} .
$$

Moreover,

$$
\sum_{i=1}^{k} S_{\ell}\left(\sigma_{i}\right) \ll_{N, E} r^{-\ell+N / 2} .
$$

Proof According to Lemma 2.10(b) there exists a non-negative smooth function $\sigma$ supported on $H_{r / 2}$ such that

$$
\int_{H} \sigma(h) d m^{\text {Haar }}(h)=1, \quad S_{\ell}(\sigma) \ll_{N} r^{-\ell+N / 2} .
$$

Since $H$ is a Riemannian manifold and $E$ is bounded, there exists a smooth partition of unity, $f_{i}: H \rightarrow \mathbb{R}, i=1, \ldots, k$, such that each $f_{i}$ is supported on a ball of radius $r / 2$ with a center $u_{i} \in E$ and

$$
\sum_{i=1}^{k} f_{i}(x)= \begin{cases}0 & \text { if } x \notin H_{r}(E) \\ 1 & \text { if } x \in H_{r / 2}(E) .\end{cases}
$$

For $i=1, \ldots, k$ define $\sigma_{i}$ by

$$
\sigma_{i}:=f_{i} * \sigma
$$

We will show that $\sigma_{1}, \ldots, \sigma_{k}$ satisfy the claim.

By definition, for $i=1, \ldots, k, \sigma_{i}$ is supported on a ball of radius $r$ and centered at a point in $E$. By Young's convolution inequality, we have

$$
S_{\ell}\left(\sigma_{i}\right) \leq S_{1,0}\left(f_{i}\right) S_{\ell}(\sigma) \ll_{N} r^{-\ell+N / 2} .
$$


For any $h \in E, h^{-1} E$ contains the identity, and so we have $h^{-1} H_{r / 2}(E) \supseteq H_{r / 2}$. Thus,

$$
\begin{aligned}
\sum_{i=1}^{k} \sigma_{i}(h) & =\sum_{i=1}^{k} \int_{H} f_{i}(x) \sigma\left(h x^{-1}\right) d m^{\text {Haar }}(x) \\
& =\int_{H} \sum_{i=1}^{k} f_{i}(x) \sigma\left(h x^{-1}\right) d m^{\text {Haar }}(x) \\
& =\int_{H_{r}(E)} \sigma\left(h x^{-1}\right) d m^{\text {Haar }}(x) \\
& =1 .
\end{aligned}
$$

If $h \notin H_{r}(E)$, then we have $h^{-1} H_{r / 2}(E) \cap H_{r / 2}=\emptyset$. Hence, the above computation yields

$$
\sum_{i=1}^{k} \sigma_{i}(h)=0 .
$$

Note that by (16), and since $f_{i}$ is a partition of unity, we may also deduce

$$
\begin{aligned}
\sum_{i=1}^{k} S_{\ell}\left(\sigma_{i}\right) & \leq S_{\ell}(\sigma) \sum_{i=1}^{k} S_{1,0}\left(f_{i}\right) \\
& =S_{\ell}(\sigma) \int_{H} \sum_{i=1}^{k} f_{i}(x) d m^{\text {Haar }}(x) \\
& \leq S_{\ell}(\sigma) m^{\text {Haar }}\left(H_{r}(E)\right) \\
& \ll N, E r^{-\ell+N / 2} .
\end{aligned}
$$

\subsection{Equidistribution results}

For the proof of the main theorems we use the equidistribution results stated below.

The following theorem was proved for $G=\mathrm{SL}_{2}(\mathbb{R})$ by Maucourant and Schapira in [12] and for $G=\operatorname{SO}(n, 1)^{\circ}$ by Mohammadi and $\mathrm{Oh}$ in [14].

Theorem 2.14 Let $\Gamma$ be geometrically finite. Fix $x \in G / \Gamma$ such that $x^{-} \in \Lambda_{r}(\Gamma)$. Then for any $\psi \in C_{c}(G / \Gamma)$ we have

$$
\lim _{T \rightarrow \infty} \frac{1}{\mu_{x}^{\mathrm{PS}}\left(B_{U}(T)\right)} \int_{B_{U}(T)} \psi(u x) d u=m^{\mathrm{BR}}(\psi) .
$$

Theorem 2.15 ([18, Theorem 1.4 and Remark 7.3]) Assume $\Gamma$ satisfies property A. For any $0<\varepsilon<1$ and $s_{0} \geq 1$, there exist $\ell=\ell(\Gamma) \in \mathbb{N}$ and $\kappa=\kappa(\Gamma, \varepsilon)>0$ satisfying: for every compact $\Omega \subset G / \Gamma$ and $\psi \in C_{c}^{\infty}(\Omega)$, there exists $c=c(\Gamma$, supp $\psi)$ such that for every $x \in G / \Gamma$ that is $\left(\varepsilon, s_{0}\right)$-Diophantine, and for all $r \gg_{\Gamma, \Omega, \varepsilon} s_{0}$,

$$
\left|\frac{1}{\mu_{x}^{\mathrm{PS}}\left(B_{U}(r)\right)} \int_{B_{U}(r)} \psi\left(u_{\boldsymbol{t}} x\right) d \boldsymbol{t}-m^{\mathrm{BR}}(\psi)\right| \leq c S_{\ell}(\psi) r^{-\kappa},
$$

where $S_{\ell}(\psi)$ is the $\ell$-Sobolev norm. 


\section{Duality between $G / \Gamma$ and $U \backslash G$}

The goal of this section is to prove the following proposition, which shows that one can use equidistribution results of $U$ orbits in $G / \Gamma$ in order to study the distribution of the points in $x \Gamma_{T}$ for $x \in U \backslash G$. This will be used to prove Theorems 1.2 and 1.9 .

Recall that for $x, y \in U \backslash G$, we defined

$$
x \star y:=\sqrt{\frac{1}{2}\left\|\Psi(x)^{-1} E_{1, n+1} \Psi(y)\right\|,}
$$

where $E_{1, n+1}$ is the $(n+1) \times(n+1)$ matrix with one in the $(1, n+1)$-entry and zeros everywhere else.

Recall the Iwasawa decomposition $G=\mathrm{SO}(n, 1)^{\circ}=U \times A \times K$. Define a continuous section by $\Psi: U \backslash G \rightarrow A K$ by

$$
\Psi(U g)=a k,
$$

where $g=u a k$ is the Iwasawa decomposition of $g$.

For $\varphi \in C_{c}(U \backslash G)$, define

$$
R_{\varphi}:=\max _{y \in \operatorname{supp} \varphi}(x \star y), \quad r_{\varphi}:=\min _{y \in \operatorname{supp} \varphi}(x \star y) .
$$

Proposition 3.1 Let $\eta>0, \Omega \subset U \backslash G$ be a compact set, $\varphi \in C(\Omega)$, and $\psi \in C\left(B_{U}(\eta)\right)$ be a non-negative function such that $\int_{U} \psi=1$. Fix $x \in U \backslash G$. Define $F \in C_{c}(G / \Gamma)$ by

$$
F(g \Gamma):=\sum_{\gamma \in \Gamma} \psi(u(g \gamma)) \varphi\left(\pi_{U}(g \gamma)\right) .
$$

Then, for some $c=c(x, \Omega)>0$,

$$
\int_{B_{U}\left(\frac{\sqrt{T}-c}{R_{\varphi}}-\eta\right)} F\left(u_{t} \Psi(x) \Gamma\right) d t \leq \sum_{\gamma \in \Gamma_{T}} \varphi(x \gamma) \leq \int_{B_{U}\left(\frac{\sqrt{T}+c}{r_{\varphi}}+\eta\right)} F\left(u_{t} \Psi(x) \Gamma\right) d t .
$$

Observe that

$$
g \Psi(U g)^{-1} \in U
$$

is by definition the $U$-component of the Iwasawa decomposition of $g$. Similarly, for any $g, h \in G$, the $U$-component of $\Psi(U h) g$ is given by

$$
\Psi(U h) g \Psi(U h g)^{-1}=\left(h \Psi(U h)^{-1}\right)^{-1}\left(h g \Psi(U h g)^{-1}\right) \in U .
$$

Hence, for any $x \in U \backslash G$ and $g \in G$, we can define $c_{x}(g):=\mathbf{t} \in \mathbb{R}^{n-1}$, where $\mathbf{t}$ is such that $\Psi(x) g \Psi(x g)^{-1}=u_{\mathbf{t}}$. Then by the actions of $U$ and $A$, we can see that this satisfies

$$
c_{e}\left(u_{\mathbf{t}} g\right)=c_{e}(g)+\mathbf{t}, \quad c_{e}\left(a_{s} g\right)=e^{s} c_{e}(g)
$$

and for any $x \in U \backslash G$,

$$
c_{x}(g)=c_{e}(\Psi(x) g) .
$$

Observe that (20) implies that

$$
c_{e}(h g)=c_{e}(h)+c_{e}(\Psi(U h) g)=c_{e}(h)+c_{U h}(g) .
$$

Note that for $g \in G$,

$$
g=u_{e}(g) \Psi(U g)
$$


That is, $u_{e}(g)$ is the $U$ component of the Iwasawa decomposition of $G$, and $\Psi(U g)$ is the $A K$ component.

For $x \in U \backslash G$ and $g \in G$, we will abuse notation and write

$$
u_{x}(g):=u_{c_{x}(g)} \text {. }
$$

Lemma 3.2 For any compact $\Omega \subset U \backslash G$ and $x \in U \backslash G$ there exist $c=c(\Omega, x)>0$ such that for any $\mathrm{xg} \in \Omega$ and $T>c$, we have

(1) If $\|g\| \leq T$, then $u_{x}(g) \in B_{U}\left(\frac{\sqrt{T}+c}{x \star x g}\right)$.

(2) If $\|g\| \geq T$, then $u_{x}(g) \notin B_{U}\left(\frac{\sqrt{T}-c}{x \star x g}\right)$.

Proof We have $g=\Psi(x)^{-1} u_{x}(g) \Psi(x g)$. For $\mathbf{t}:=c_{x}(g)$ we get

$$
\begin{aligned}
g & =\Psi(x)^{-1} u_{x}(g) \Psi(x g) \\
& =\Psi(x)^{-1}\left(I+\left(\begin{array}{lll}
0 & \mathbf{t} & 0 \\
0 & 0 & \mathbf{t}^{T} \\
0 & 0 & 0
\end{array}\right)+\|\mathbf{t}\|^{2} E_{1, n+1}\right) \Psi(x g)
\end{aligned}
$$

Denote

$$
\begin{aligned}
& c_{1}:=\max _{y \in \Omega}\left\{\left\|\Psi(x)^{-1} \Psi(y)\right\|\right\}, \\
& c_{2}:=\max _{y \in \Omega,\|\mathbf{t}\| \leq 1}\left\{\left\|\Psi(x)^{-1}\left(\begin{array}{lll}
0 & \mathbf{t} & 0 \\
0 & 0 & \mathbf{t}^{T} \\
0 & 0 & 0
\end{array}\right) \Psi(y)\right\|\right\} .
\end{aligned}
$$

Then, $c_{1}$ and $c_{2}$ are functions of $x$ and $\Omega$. By the triangle inequality,

$$
\begin{aligned}
\|g\| & \leq\|\mathbf{t}\|^{2}(x \star x g)^{2}+\left\|\Psi(x)^{-1} \Psi(x g)\right\|+\left\|\Psi(x)^{-1}\left(\begin{array}{ccc}
0 & \mathbf{t} & 0 \\
0 & 0 & \mathbf{t}^{T} \\
0 & 0 & 0
\end{array}\right) \Psi(x g)\right\| \\
& \leq\|\mathbf{t}\|^{2}(x \star x g)^{2}+c_{1}+c_{2}\|\mathbf{t}\| .
\end{aligned}
$$

In a similar way

$$
\begin{aligned}
\|g\| & \geq\|\mathbf{t}\|^{2}(x \star x g)^{2}-\left\|\Psi(x)^{-1} \Psi(x g)\right\|-\left\|\Psi(x)^{-1}\left(\begin{array}{ccc}
0 & \mathbf{t} & 0 \\
0 & 0 & \mathbf{t}^{T} \\
0 & 0 & 0
\end{array}\right) \Psi(x g)\right\| \\
& \geq\|\mathbf{t}\|^{2}(x \star x g)^{2}-c_{1}-c_{2}\|\mathbf{t}\| .
\end{aligned}
$$

We conclude that for any $g \in \Omega$,

$$
\left|\|g\|-(x \star x g)^{2}\|\mathbf{t}\|^{2}\right| \leq c_{1}+c_{2}\|\mathbf{t}\| .
$$

Assume $\|g\| \geq T \geq c_{1}$. Then, by (22)

$$
0 \leq(x \star x g)^{2}\|\mathbf{t}\|^{2}+c_{2}\|\mathbf{t}\|+\left(c_{1}-T\right) .
$$

Using the quadratic formula, we may deduce that the right hand side of the above equation is equal to zero when

$$
\|\mathbf{t}\|=\frac{-c_{2} \pm \sqrt{c_{2}^{2}+4\left(T-c_{1}\right)(x \star x g)^{2}}}{2(x \star x g)^{2}}
$$


Since $(x \star x g)^{2}$ and $\|t\|$ are non-negative, it follows that

$$
\|\mathbf{t}\| \geq \frac{-c_{2}+\sqrt{c_{2}^{2}+4\left(T-c_{1}\right)(x \star x g)^{2}}}{2(x \star x g)^{2}}
$$

Using the inequality $\sqrt{a \pm b} \geq \sqrt{a}-\sqrt{b}$, we arrive at

$$
\|\mathbf{t}\| \geq \frac{\sqrt{T}}{x \star x g}-\frac{c_{2}+c_{1} x \star x g}{(x \star x g)^{2}}
$$

A similar computation shows that $\|g\| \leq T$ implies

$$
\|\mathbf{t}\| \leq \frac{\sqrt{T}}{x \star x g}+\frac{c_{2}+c_{1} x \star x g}{(x \star x g)^{2}} .
$$

Letting $c$ be the maximum of $\frac{c_{2}+c_{1} x \star x g}{(x \star x g)^{2}}$ for $g \in \Omega$ completes the proof.

Lemma 3.3 Let $\varphi \in C_{c}(U \backslash G)$ and suppose that $\psi \in C_{c}(U)$ satisfies

$$
\int_{U} \psi=1
$$

For $g \in G$, define

$$
f(g)=\psi(u(g)) \varphi\left(\pi_{U}(g)\right)
$$

Then for every $g \in G$,

$$
\varphi\left(\pi_{U}(g)\right)=\int_{\operatorname{supp}(\psi) u(g)^{-1}} f\left(u_{t} g\right) d t .
$$

Proof By the definition of $\psi$,

$$
\begin{aligned}
\varphi\left(\pi_{U}(g)\right) & =\varphi\left(\pi_{U}(g)\right) \int_{\operatorname{supp}(\psi)} \psi\left(u_{\mathbf{t}}\right) d \mathbf{t} \\
& =\varphi\left(\pi_{U}(g)\right) \int_{u(g)^{-1} \operatorname{supp}(\psi)} \psi\left(u(g) u_{\mathbf{t}}\right) d \mathbf{t} .
\end{aligned}
$$

Since $\pi_{U}\left(u_{t} g\right)=\pi_{U}(g)$, we have

$$
\begin{aligned}
\varphi\left(\pi_{U}(g)\right) & =\int_{u(g)^{-1} \operatorname{supp}(\psi)} \psi\left(u\left(u_{\mathbf{t}} g\right)\right) \varphi\left(\pi_{U}\left(u_{\mathbf{t}} g\right)\right) d \mathbf{t} \\
& =\int_{u(g)^{-1} \operatorname{supp}(\psi)} f\left(u_{\mathbf{t}} g\right) d \mathbf{t}
\end{aligned}
$$

We are now ready to prove Proposition 3.1.

Proof of Proposition 3.1 Without loss of generality, we may assume that $\varphi \geq 0$. Define $f$ : $G \rightarrow \mathbb{R}$ by

$$
f(g)=\psi(u(g)) \varphi\left(\pi_{U}(g)\right) .
$$

By Lemma 3.3, for every $g \in G$,

$$
\varphi\left(\pi_{U}(g)\right)=\int_{u(g)^{-1} B_{U}(\eta)} f\left(u_{\mathbf{t}} g\right) d \mathbf{t} .
$$


By Lemma 3.2, there exist $c>0$ depending on $\Omega$ and $x$ such that for all $T \geq c$, if $\gamma \in \Gamma_{T}$ and $x \gamma \in \Omega$, then

$$
u_{x}(\gamma)^{-1} B_{U}(\eta) \subseteq B_{U}\left(\frac{\sqrt{T}+c}{x \star x \gamma}+\eta\right) .
$$

Observe also that since $\operatorname{supp}(\psi) \subseteq B_{U}(\eta)$, if $u_{\mathbf{t}} \notin u_{x}(\gamma)^{-1} B_{U}(\eta)$, then

$$
f\left(u_{\mathbf{t}} \Psi(x) \gamma\right)=\psi\left(u_{\mathbf{t}} u(\Psi(x) \gamma)\right) \varphi\left(\pi_{U}(\Psi(x) \gamma)\right)=0 .
$$

Thus, using (21) and Lemma 3.3, for $\gamma \in \Gamma_{T}$ with $x \gamma \in \Omega$, we have that

$$
\begin{aligned}
\varphi(x \gamma) & =\int_{u(\Psi(x) \gamma)^{-1} B_{U}(\eta)} f\left(u_{\mathbf{t}} \Psi(x) \gamma\right) d \mathbf{t} \\
& =\int_{u_{x}(\gamma)^{-1} B_{U}(\eta)} f\left(u_{\mathbf{t}} \Psi(x) \gamma\right) d \mathbf{t} \\
& =\int_{B_{U}\left(\frac{\sqrt{T}+c}{x \star x \gamma}+\eta\right)} f\left(u_{\mathbf{t}} \Psi(x) \gamma\right) d \mathbf{t} .
\end{aligned}
$$

Note that

$$
F(g \Gamma):=\sum_{\gamma \in \Gamma} f(g \gamma)
$$

Thus, from (23), for $r=r_{\varphi}:=\min _{y \in \operatorname{supp} \varphi}(x \star y)$, we obtain

$$
\begin{aligned}
\sum_{\gamma \in \Gamma_{T}} \varphi(x \gamma) & \leq \sum_{\gamma \in \Gamma_{T}} \int_{B_{U}\left(\frac{\sqrt{T}+c}{r}+\eta\right)} f\left(u_{\mathbf{t}} \Psi(x) \gamma\right) d \mathbf{t} \\
& \leq \int_{B_{U}\left(\frac{\sqrt{T}+c}{r}+\eta\right)} F\left(u_{\mathbf{t}} \Psi(x) \Gamma\right) d \mathbf{t} .
\end{aligned}
$$

To obtain a lower bound, we must control the terms arising from $\gamma \in \Gamma \backslash \Gamma_{T}$ in the definition of $F$. Note that by Lemma 3.2, if $\gamma \in\left(\Gamma \backslash \Gamma_{T}\right)$ and $x \gamma \in \Omega$, then we see that

$$
u_{x}(\gamma)^{-1} B_{U}(\eta) \cap B_{U}\left(\frac{\sqrt{T}-c}{x \star x \gamma}-\eta\right)=\emptyset .
$$

Thus, by (23), we obtain

$$
\begin{aligned}
\sum_{\gamma \in \Gamma_{T}} \varphi(x \gamma) & =\sum_{\gamma \in \Gamma_{T}} \int_{B_{U}\left(\frac{\sqrt{T}-c}{x \star x g}-\eta\right)} f\left(u_{\mathbf{t}} \Psi(x) \gamma\right) d \mathbf{t} \\
& =\sum_{\gamma \in \Gamma} \int_{B_{U}\left(\frac{\sqrt{T}-c}{x \star x \gamma}-\eta\right)} f\left(u_{\mathbf{t}} \Psi(x) \gamma\right) d \mathbf{t}
\end{aligned}
$$

Now, similarly to the above, we conclude that

$$
\sum_{\gamma \in \Gamma_{T}} \varphi(x \gamma) \geq \sum_{\gamma \in \Gamma} \int_{B_{U}\left(\frac{\sqrt{T}-c}{R}-\eta\right)} f\left(u_{\mathbf{t}} \Psi(x) \gamma\right) d \mathbf{t},
$$

where $R=R_{\varphi}:=\max _{y \in \operatorname{supp} \varphi}(x \star y)$, Then, the claim follows from the definition of $F,(26)$. 
Lemma 3.4 Let $\varphi \in C_{c}(U \backslash G)$ and $F$ be as defined in Proposition 3.1. Then,

$$
m^{\mathrm{BR}}(F)=\int_{P} \varphi\left(\pi_{U}(p)\right) d v(p)
$$

Proof By the definition of $F$ and the assumption that $\int_{U} \psi=1$, by the product structure of the BR measure in (14), we obtain

$$
\begin{aligned}
m^{\mathrm{BR}}(F) & =\int_{G} \psi(u(g)) \varphi\left(\pi_{U}(g)\right) d \tilde{m}^{\mathrm{BR}}(g) \\
& =\int_{P} \int_{U} \psi\left(u_{\mathbf{t}} u(p)\right) \varphi\left(\pi_{U}(p)\right) d \mathbf{t} d \nu(p) \\
& =\int_{P} \varphi\left(\pi_{U}(p)\right) d \nu(p) .
\end{aligned}
$$

For a set $H \subseteq G$, let

$$
B(H, r)=\{g \in G: d(g, H) \leq r\},
$$

where $d$ is the Riemannian metric on $G$. That is, $B(H, r)$ is the $r$-thickening of $H$ with respect to $d$. For $h \in G$, we denote $B(\{h\}, r)$ by $B(h, r)$ (in this case we get the Riemannian ball around the point $h$ ).

For $H \subseteq G$, denote by

$$
\operatorname{inj}(H)
$$

the infimum over all $r>0$ satisfying that for every $h \in H$,

$$
\left.\pi_{\Gamma}\right|_{B(h, r)}: B(h, r) \rightarrow G / \Gamma
$$

is injective.

In the later sections, we will require a partition of $\varphi$, say into $\varphi_{1}, \ldots, \varphi_{k}$ so that for each $i, R_{\varphi_{i}}$ and $r_{\varphi_{i}}$ are close.

Lemma 3.5 Fix $x \in U \backslash G$. For a compact set $H \subseteq G$, there exists $0<\eta_{0}=\eta(H)<\operatorname{inj}(H)$, $\beta=\beta(H)>1$ so that for any $0<\eta<\eta_{0}$ and $\varphi \in C_{c}(U \backslash G)$ with $\operatorname{supp} \varphi \subset \pi_{U}(B(h, \eta))$ for some $h \in H$, we have that

$$
\frac{R_{\varphi}}{r_{\varphi}}-1 \leq\left\|\Psi(x)^{-1}\right\| \beta \eta
$$

Proof Since $B(H, 1)$ is a compact set, by [4, Lemma 9.12], there exist constants $0<\eta_{0}=$ $\eta(H)<\operatorname{inj}(H), \beta=\beta(H)>1$, such that $\eta_{0}<1$ and for all $g, h \in B(H, 1)$ with $d(g, h) \leq \eta_{0}$,

$$
\beta^{-1}\|g-h\| \leq d(g, h) \leq \beta\|g-h\| .
$$

Therefore, for any $h \in H$ and $0<\eta<\eta_{0}$, we have

$$
B(h, \eta) \subseteq\{g \in G:\|g-h\| \leq \beta \eta\} .
$$

Note that for any $g \in G$,

$$
E_{1, n+1} \Psi\left(\pi_{U}(g)\right)=E_{1, n+1} g .
$$

Thus, if $\|g-h\|<\beta \eta$, then 


$$
\begin{aligned}
\left\|\Psi(x)^{-1} E_{1, n+1} \Psi\left(\pi_{U}(g)\right)\right\| & =\left\|\Psi(x)^{-1} E_{1, n+1} g\right\| \\
& \leq\left\|\Psi(x)^{-1} E_{1, n+1} h\right\|+\left\|\Psi(x)^{-1} E_{1, n+1}(g-h)\right\| \\
& \leq\left\|\Psi(x)^{-1} E_{1, n+1} \Psi\left(\pi_{U}(h)\right)\right\|+\beta \eta\left\|\Psi(x)^{-1}\right\|,
\end{aligned}
$$

and similarly

$$
\left\|\Psi(x)^{-1} E_{1, n+1} \Psi\left(\pi_{U}(g)\right)\right\| \geq\left\|\Psi(x)^{-1} E_{1, n+1} \Psi\left(\pi_{U}(h)\right)\right\|-\beta \eta\left\|\Psi(x)^{-1}\right\| .
$$

Thus, it follows from (17) that for

$$
R=\max _{y \in \pi_{U}(B(h, \eta))}(x \star y), \quad r=\min _{y \in \pi_{U}(B(h, \eta))}(x \star y),
$$

we have

$$
R-r \leq 2 \beta\left\|\Psi(x)^{-1}\right\| \eta .
$$

Since $r$ is bounded below by a constant depending on $H$, this implies that

$$
\left(\frac{R}{r}\right)-1 \ll_{H}\left\|\Psi(x)^{-1}\right\| \eta
$$

Corollary 3.6 Fix $x \in U \backslash G$ and $\varphi \in C_{c}(U \backslash G)$. Let $\eta_{0}=\eta_{0}(\Psi(\operatorname{supp} \varphi))$ be as in Lemma 3.5. For any $0<\eta<\eta_{0}$, there exist some $k$ and $\varphi_{1}, \ldots, \varphi_{k} \in C_{c}(U \backslash G)$ so that

$$
\sum_{i=1}^{k} \varphi_{i}=\varphi \quad \text { and } \frac{R_{\varphi_{i}}}{r_{\varphi_{i}}}-1 \ll_{\Gamma, \operatorname{supp} \varphi} \eta
$$

Moreover, if $\varphi \in C_{c}^{\infty}(U \backslash G)$, then we also have $\varphi_{i} \in C_{c}^{\infty}(U \backslash G)$, and that for any $\ell^{\prime}>0$, there exists $\ell>\ell^{\prime}$ satisfying

$$
\sum_{i=1}^{k} S_{\ell^{\prime}}\left(\varphi_{i}\right) \ll \ell, \operatorname{supp} \varphi \eta^{-\ell+n(n+1) / 4} S_{\ell}(\varphi) .
$$

Proof For the first case (only assuming $\varphi \in C_{c}(U \backslash G)$ ), cover $\Psi$ (supp $\varphi$ ) with balls of radius $\eta$, and let $\sigma_{1}, \ldots, \sigma_{k}$ be a partition of unity subordinate to this cover. Defining

$$
\varphi_{i}=\varphi \cdot\left(\sigma_{i} \circ \Psi\right)
$$

yields functions with the desired property, by Lemma 3.5.

Now, assume that $\varphi \in C_{c}^{\infty}(U \backslash G)$, and let $\ell>\ell^{\prime}$ satisfy the conclusion of Lemma 2.12 for $\ell^{\prime}$. We must be more careful in order to control Sobolev norms. By Lemma 2.13, for $0<\eta \leq \eta_{0}$, there exist $h_{1}, \ldots, h_{k} \in \Psi(\operatorname{supp} \varphi)$ and $\sigma_{1}, \ldots, \sigma_{k} \in C_{c}^{\infty}\left(B\left(h_{i}, \eta\right)\right)$ with

$$
\sum_{i=1}^{k} \sigma_{i}=1 \text { on } \Psi(\operatorname{supp} \varphi) \text { and }=0 \text { outside } B(\Psi(\operatorname{supp} \varphi), \eta)
$$

and such that

$$
\sum_{i=1}^{k} S_{\ell}\left(\sigma_{i}\right) \ll_{n, \operatorname{supp} \varphi} \eta^{-\ell+n(n+1) / 4}
$$

Define

$$
\varphi_{i}=\varphi \cdot\left(\sigma_{i} \circ \Psi\right) .
$$


Then, by Lemma 3.5,

$$
\frac{R_{\varphi_{i}}}{r_{\varphi_{i}}}-1 \ll \operatorname{supp} \varphi\left\|\Psi(x)^{-1}\right\| \eta
$$

Since $\Psi$ is smooth and $\ell>\ell^{\prime}$, by Lemmas 2.11 and 2.12,

$$
\begin{aligned}
S_{\ell^{\prime}}\left(\varphi_{i}\right) & \ll_{\ell} S_{\ell^{\prime}}(\varphi) S_{\ell^{\prime}}\left(\sigma_{i} \circ \Psi\right) \\
& \ll_{\ell, \Psi} S_{\ell}(\varphi) S_{\ell}\left(\sigma_{i}\right) .
\end{aligned}
$$

From (31) and (32), we conclude that

$$
\sum_{i=1}^{k} S_{\ell^{\prime}}\left(\varphi_{i}\right) \ll \ell, n, \operatorname{supp} \varphi, \Psi \eta^{-\ell+n(n+1) / 4} S_{\ell}(\varphi) .
$$

\section{Proof of Theorem 1.2}

In this section, we prove Theorem 1.2, which is restated below for convenience.

Theorem 4.1 Let $\Gamma$ be geometrically finite. For any $\varphi \in C_{c}(U \backslash G)$ and every $x \in U \backslash G$ such that $\Psi(x)^{-} \in \Lambda_{r}(\Gamma)$,

$$
\sum_{\gamma \in \Gamma_{T}} \varphi(x \gamma) \sim I(\varphi, T, x) .
$$

We will need the following lemma. Theorem 1.2 will then follow by a partition of unity argument.

Lemma 4.2 Let $\varphi \in C_{c}(U \backslash G)$ and let $x \in U \backslash G$ be such that $\Psi(x)^{-} \in \Lambda_{r}(\Gamma)$. Let $R=R_{\varphi}$ and $r=r_{\varphi}$ be as in (18). Let $\eta>0$, and suppose that $\frac{R}{r}<1+\eta$ and that $B_{U}(\eta) \Psi(\operatorname{supp} \varphi)$ injects into $G / \Gamma$.

Then for any $\varepsilon>0$, there exists $T_{1}=T_{1}(x, \eta, \varphi)>0$ such that for all $T \geq T_{1}$,

$$
\begin{aligned}
& \left|\sum_{\gamma \in \Gamma_{T}} \varphi(x \gamma)-\int_{P} \mu_{\Psi(x) \Gamma}^{\mathrm{PS}}\left(B_{U}\left(\frac{\sqrt{T}}{x \star \pi_{U}(p)}\right)\right) \varphi\left(\pi_{U}(p)\right) d \nu(p)\right| \\
& \quad \ll \Gamma, x \mu_{\Psi(x) \Gamma}^{\mathrm{PS}}\left(B_{U}\left(\frac{\sqrt{T}}{r}\right)\right)\left[\left(\eta+\frac{c+1}{\sqrt{T}}\right)^{\alpha} \int_{P} \varphi\left(\pi_{U}(p)\right) d \nu(p)+\varepsilon\right],
\end{aligned}
$$

where $\alpha=\alpha(\Gamma)$ is from Lemma 2.9, and $c=c(x, \operatorname{supp} \varphi)>0$ is as in Proposition 3.1.

Remark 4.3 Note that $T_{1}$ depends on $\eta$ through a non-canonical choice of bump function $\psi$, as seen in the proof. When we apply this lemma to a partition of unity, the same $\psi$ will be used for each part.

Proof Let $\psi \in C\left(B_{U}(\eta)\right)$ be a non-negative function such that $\int_{U} \psi=1$. Let $F$ and $c=c(x, \operatorname{supp} \varphi)>0$ be as in the statement of Proposition 3.1 for this $\psi$, and let $\varepsilon>0$.

By Theorem 2.14, there exists $T_{1}=T_{1}(x, \psi, \varphi)$ such that for $T \geq T_{1}$,

$$
\mu_{\Psi(x) \Gamma}^{\mathrm{PS}}\left(B_{U}\left(\frac{\sqrt{T}-c}{R}-\eta\right)\right)\left(m^{\mathrm{BR}}(F)-\varepsilon\right)
$$




$$
\begin{aligned}
& \leq \sum_{\gamma \in \Gamma_{T}} \varphi(x \gamma) \\
& \leq \mu_{\Psi(x) \Gamma}^{\mathrm{PS}}\left(B_{U}\left(\frac{\sqrt{T}+c}{r}+\eta\right)\right)\left(m^{\mathrm{BR}}(F)+\varepsilon\right) .
\end{aligned}
$$

Let $y \in \operatorname{supp}(\varphi)$. By combining the above with Lemma 2.9 (using $R, r$, and $\ell=x \star y$ ), we see that there exist constants $c_{0}=c_{0}(\Gamma, x)$ and $T_{2}=T_{2}(\Gamma, x, \operatorname{supp} \varphi)>0$ such that for $T \geq T_{2}$

$$
\begin{aligned}
& \left(1-c_{0}\left(\eta+\frac{c+1}{\sqrt{T}}\right)^{\alpha}\right)\left(m^{\mathrm{BR}}(F)-\varepsilon\right) \\
& \leq \frac{1}{\mu_{\Psi(x) \Gamma}^{\mathrm{PS}}\left(B_{U}\left(\frac{\sqrt{T}}{x \star y}\right)\right)} \sum_{\gamma \in \Gamma_{T}} \varphi(x \gamma) \\
& \leq\left(1+c_{0}\left(\eta+\frac{c+1}{\sqrt{T}}\right)^{\alpha}\right)\left(m^{\mathrm{BR}}(F)+\varepsilon\right) .
\end{aligned}
$$

By Lemma 3.4, $m^{\mathrm{BR}}(F)=\int_{P} \varphi\left(\pi_{U}(p)\right) d \nu(p)$, and so by (36), for any $y \in \operatorname{supp} \varphi$, we obtain that

$$
\begin{aligned}
& \left|\frac{1}{\mu_{\Psi(x) \Gamma}^{\mathrm{PS}}\left(B_{U}\left(\frac{\sqrt{T}}{x \star y}\right)\right)} \sum_{\gamma \in \Gamma_{T}} \varphi(x \gamma)-\int_{P} \varphi\left(\pi_{U}(p)\right) d \nu(p)\right| \\
& \quad \ll \Gamma\left(\eta+\frac{c+1}{\sqrt{T}}\right)^{\alpha} \int_{P} \varphi\left(\pi_{U}(p)\right) d \nu(p)+\varepsilon .
\end{aligned}
$$

Since the above holds for any $y \in \operatorname{supp} \varphi$, by bounding

$$
\begin{aligned}
& \sum_{\gamma \in \Gamma_{T}} \varphi(x \gamma)-\mu_{\Psi(x) \Gamma}^{\mathrm{PS}}\left(B_{U}\left(\frac{\sqrt{T}}{r}\right)\right) \int_{P} \varphi\left(\pi_{U}(p)\right) d \nu(p) . \\
& \leq \sum_{\gamma \in \Gamma_{T}} \varphi(x \gamma)-\int_{P} \mu_{\Psi(x) \Gamma}^{\mathrm{PS}}\left(B_{U}\left(\frac{\sqrt{T}}{x \star \pi_{U}(p)}\right)\right) \varphi\left(\pi_{U}(p)\right) d \nu(p) \\
& \leq \sum_{\gamma \in \Gamma_{T}} \varphi(x \gamma)-\mu_{\Psi(x) \Gamma}^{\mathrm{PS}}\left(B_{U}\left(\frac{\sqrt{T}}{R}\right)\right) \int_{P} \varphi\left(\pi_{U}(p)\right) d \nu(p),
\end{aligned}
$$

we obtain

$$
\begin{aligned}
& \left|\sum_{\gamma \in \Gamma_{T}} \varphi(x \gamma)-\int_{P} \mu_{\Psi(x) \Gamma}^{\mathrm{PS}}\left(B_{U}\left(\frac{\sqrt{T}}{x \star \pi_{U}(p)}\right)\right) \varphi\left(\pi_{U}(p)\right) d \nu(p)\right| \\
& \quad \ll \Gamma, x \mu_{\Psi(x) \Gamma}^{\mathrm{PS}}\left(B_{U}\left(\frac{\sqrt{T}}{r}\right)\right)\left[\left(\eta+\frac{c+1}{\sqrt{T}}\right)^{\alpha} \int_{P} \varphi\left(\pi_{U}(p)\right) d \nu(p)+\varepsilon\right] .
\end{aligned}
$$

We are now ready to prove Theorem 1.2. 
Proof of Theorem 1.2 By Corollary 3.6, there exists $\eta_{0}=\eta_{0}(\Psi(\operatorname{supp} \varphi))>0$ so that for every $0<\eta<\eta_{0}$, there exists $\left\{\varphi_{i}: 1 \leq i \leq k\right\}$ that are a partition of $\varphi$, i.e.,

$$
\varphi=\sum_{i=1}^{k} \varphi_{i}
$$

so that all the $\varphi_{i}$ are supported on a small neighborhood of $\operatorname{supp} \varphi$, which we denote by $B$, and each $\varphi_{i}$ satisfies the assumptions of Lemma 4.2.

For any $1 \leq i \leq k$ let,

$$
R_{i}=R_{\varphi_{i}}, \quad r_{i}=r_{\varphi_{i}}
$$

as in (18).

Note that

$$
R:=\max _{y \in B}(x \star y), \quad r:=\min _{y \in B}(x \star y)
$$

satisfy $R \geq R_{i} \geq r_{i} \geq r$ for any $i$.

Fix $\varepsilon>0$. By Lemma 4.2, there exists $T_{1}>0$ (depending on the $\varphi_{i}$ 's, $x, \eta$, and $\varepsilon$ ) such that for all $T \geq T_{1}$ and for each $i$,

$$
\begin{aligned}
& \left|\sum_{\gamma \in \Gamma_{T}} \varphi_{i}(x \gamma)-\int_{P} \mu_{\Psi(x) \Gamma}^{\mathrm{PS}}\left(B_{U}\left(\frac{\sqrt{T}}{x \star \pi_{U}(p)}\right)\right) \varphi_{i}\left(\pi_{U}(p)\right) d v(p)\right| \\
& \quad \ll \Gamma, x \mu_{\Psi(x) \Gamma}^{\mathrm{PS}}\left(B_{U}\left(\frac{\sqrt{T}}{r}\right)\right)\left[\left(\eta+\frac{c+1}{\sqrt{T}}\right)^{\alpha} \int_{P} \varphi_{i}\left(\pi_{U}(p)\right) d v(p)+\frac{\varepsilon}{k}\right] .
\end{aligned}
$$

Summing over $i$, we obtain

$$
\begin{aligned}
& \left|\sum_{\gamma \in \Gamma_{T}} \varphi(x \gamma)-\int_{P} \mu_{\Psi(x) \Gamma}^{\mathrm{PS}}\left(B_{U}\left(\frac{\sqrt{T}}{x \star \pi_{U}(p)}\right)\right) \varphi\left(\pi_{U}(p)\right) d \nu(p)\right| \\
& \quad \ll \Gamma, x \mu_{\Psi(x) \Gamma}^{\mathrm{PS}}\left(B_{U}\left(\frac{\sqrt{T}}{r}\right)\right)\left[\left(\eta+\frac{c+1}{\sqrt{T}}\right)^{\alpha} \int_{P} \varphi\left(\pi_{U}(p)\right) d v(p)+\varepsilon\right] .
\end{aligned}
$$

Recall that

$$
I(\varphi, T, x):=\int_{P} \mu_{\Psi(x) \Gamma}^{\mathrm{PS}}\left(B_{U}\left(\frac{\sqrt{T}}{x \star \pi_{U}(p)}\right)\right) \varphi\left(\pi_{U}(p)\right) d v(p) .
$$

By Lemma 2.4, there exists $\sigma=\sigma(\Gamma)>0$ so that for any $y \in \operatorname{supp} \varphi$,

$$
\frac{\mu_{\Psi(x) \Gamma}^{\mathrm{PS}}\left(B_{U}\left(\frac{\sqrt{T}}{r}\right)\right)}{\mu_{\Psi(x) \Gamma}^{\mathrm{PS}}\left(B_{U}\left(\frac{\sqrt{T}}{x \star y}\right)\right)} \ll_{\Gamma}\left(\frac{R}{r}\right)^{\sigma} .
$$

Thus, from (37), we obtain

$$
\left|\frac{\sum_{\gamma \in \Gamma_{T}} \varphi(x \gamma)}{I(\varphi, T, x)}-1\right|
$$




$$
\ll_{\Gamma}\left(\frac{R}{r}\right)^{\sigma} v\left(\varphi \circ \pi_{U}\right)^{-1}\left[\left(\eta+\frac{c+1}{\sqrt{T}}\right)^{\alpha} \int_{P} \varphi\left(\pi_{U}(p)\right) d v(p)+\varepsilon\right] .
$$

Since $\eta$ and $\varepsilon$ can be chosen arbitrarily small, the claim follows.

We will now deduce Corollary 1.3 using the shadow lemma, Proposition 2.1.

Proof of Corollary 1.3 Since $\Psi(x)^{-} \in \Lambda_{r}(\Gamma)$, there exists $r=r(x) \geq 0$ such that

$$
B_{U}(r) \Psi(x) \Gamma \cap \operatorname{supp} m^{\mathrm{BMS}} \neq \emptyset .
$$

Let $w \in B_{U}(r) \Psi(x) \Gamma \cap \operatorname{supp} m^{\mathrm{BMS}} \subseteq G / \Gamma$. Then for any $T \geq 0$,

$$
\mu_{w}^{\mathrm{PS}}\left(B_{U}(T-r)\right) \leq \mu_{\Psi(x) \Gamma}^{\mathrm{PS}}\left(B_{U}(T)\right) \leq \mu_{w}^{\mathrm{PS}}\left(B_{U}(T+r)\right) .
$$

Thus, by Proposition 2.1, there exists $\lambda=\lambda(\Gamma)>1$ such that for all $T \geq 0$,

$$
\lambda^{-1}(T-r)^{\delta_{\Gamma}} \leq \mu_{\Psi(x) \Gamma}^{\mathrm{PS}}\left(B_{U}(T)\right) \leq \lambda(T+r)^{\delta_{\Gamma}} .
$$

For every $y \in \operatorname{supp} \varphi$, we therefore have that for all $T \geq 2 r$,

$$
\frac{T^{\delta_{\Gamma} / 2}}{(x \star y)^{\delta_{\Gamma}}} \ll \Gamma, x \mu_{\Psi(x) \Gamma}^{\mathrm{PS}}\left(\frac{\sqrt{T}}{x \star y}\right) \ll \Gamma, x \frac{T^{\delta_{\Gamma} / 2}}{(x \star y)^{\delta_{\Gamma}}} .
$$

By Theorem 1.2, there exists $T_{0}=T_{0}(x, \varphi)$ such that for $T \geq T_{0}$,

$$
\left|\frac{\sum_{\gamma \in \Gamma_{T}} \varphi(x \gamma)}{I(\varphi, T, x)}-1\right| \leq 1 / 2 .
$$

Then

$$
\frac{1}{\mu_{\Psi(x) \Gamma}^{\mathrm{PS}}\left(\frac{\sqrt{T}}{x \star y}\right)} \sum_{\gamma \in \Gamma_{T}} \varphi(x \gamma) \leq \frac{2}{\mu_{\Psi(x) \Gamma}^{\mathrm{PS}}\left(\frac{\sqrt{T}}{x \star y}\right)} I(\varphi, T, x)
$$

so by (38), we obtain

$$
\begin{aligned}
\frac{1}{T^{\delta_{\Gamma} / 2}} \sum_{\gamma \in \Gamma_{T}} \varphi(x \gamma) & \ll \Gamma, x \frac{1}{T^{\delta_{\Gamma} / 2}} \int_{P} \mu_{\Psi(x) \Gamma}^{\mathrm{PS}}\left(\frac{\sqrt{T}}{x \star \pi_{U}(p)}\right) \varphi\left(\pi_{U}(p)\right) d \nu(p) \\
& \ll \Gamma, x \frac{1}{T^{\delta_{\Gamma} / 2}} \int_{P} \frac{T^{\delta_{\Gamma} / 2}}{\left(x \star \pi_{U}(p)\right)^{\delta_{\Gamma}}} \varphi\left(\pi_{U}(p)\right) d \nu(p) \\
& \ll \Gamma, x \int_{P} \frac{\varphi\left(\pi_{U}(p)\right)}{\left(x \star \pi_{U}(p)\right)^{\delta_{\Gamma}}} d \nu(p) .
\end{aligned}
$$

The lower bound is very similar.

\section{A small support "Ergodic Theorem"}

In this section, we prove an ergodic-theorem type statement for functions with small support. This result will be used in the next chapter to prove Theorem 1.9.

Recall that for $x \in U \backslash G$ and a compact set $H \subset U \backslash G$, let

$$
\mathcal{R}(H, x):=\max _{y, z \in H} \frac{x \star y}{x \star z} .
$$


Theorem 5.1 Let $\Gamma$ satisfy property $A$. There exists $\ell=\ell(\Gamma) \in \mathbb{N}$ so that for any $0<\varepsilon<1$, there exists $\kappa=\kappa(\Gamma, \varepsilon)$ satisfying: for every $x \in U \backslash G$ such that $\Psi(x) \Gamma$ is $\varepsilon$-Diophantine and every compact $\Omega \subset G$, there exists $T_{0}=T_{0}(x, \Omega)$ so that for every $T \geq T_{0}$, there exists $\eta=\eta(T, \ell, \kappa, n, \Omega)>0$ such that if $\varphi \in C_{c}^{\infty}(U \backslash G)$ with $\Psi(\operatorname{supp} \varphi) \subseteq \Omega$ and satisfies $\mathcal{R}(\operatorname{supp} \varphi, x)-1<\eta$, then for every $y \in \operatorname{supp} \varphi$,

$$
\left|\frac{1}{\mu_{\Psi(x) \Gamma}^{\mathrm{PS}}\left(B_{U}\left(\frac{\sqrt{T}}{x \star y}\right)\right)} \sum_{\gamma \in \Gamma_{T}} \varphi(x \gamma)-\int_{P} \varphi\left(\pi_{U}(p)\right) d \nu(p)\right| \ll_{\Gamma, \Omega, x} S_{\ell}(\varphi) T^{-\kappa} .
$$

Proof Fix $x \in U \backslash G$ such that $\Psi(x) \Gamma$ is $\varepsilon$-Diophantine. Let $0<\eta_{1}=\eta_{1}(\Omega)<1$ be such that for all $g \in \Omega$,

$$
\left.\pi_{\Gamma}\right|_{B\left(g, \eta_{1}\right)}: B\left(g, \eta_{1}\right) \rightarrow G / \Gamma
$$

is injective, where $B\left(g, \eta_{1}\right)=\left\{h \in G:\|g-h\| \leq \eta_{1}\right\}$. Let $0<\eta<\eta_{1}$. Then if $\Psi(\operatorname{supp} \varphi) \subset \Omega \subset G$, we have that

$$
B:=B_{U}(\eta) \Psi(\operatorname{supp} \varphi)
$$

injects into $G / \Gamma$. Let $R=R_{\varphi}, r=r_{\varphi}$ as in (18). We are assuming that

$$
\mathcal{R}(\operatorname{supp} \varphi, x)-1=\frac{R}{r}-1<\eta .
$$

We will find $T_{0}=T_{0}(x, \Omega)$ as in the statement of the theorem, and choose $\eta$ depending on $T \geq T_{0}$ later.

According to Lemma 2.10(2), there exists $\psi: U \rightarrow \mathbb{R}$ such that $\operatorname{supp} \psi=B_{U}(\eta)$ and

$$
\int_{U} \psi=1, \quad S_{\ell}(\psi) \ll \eta^{-\ell+n-1} .
$$

We can now use Proposition 3.1 with the above $\psi$ and $\varphi$ to get an expression that we can estimate using the effective equidistribution theorem, Theorem 2.15.

Let $F$ and $c=c(\Omega, x)$ be as in Proposition 3.1 for $\psi, \varphi$. There exists $\ell, \kappa^{\prime}, c_{2}=$ $c_{2}(\Gamma, \operatorname{supp} \psi, x)$ as in the statement of Theorem 2.15 and $T_{1}=T_{1}(x, \Omega) \geq c$ such that for all $T \geq T_{1}$,

$$
\begin{aligned}
& \mu_{\Psi(x) \Gamma}^{\mathrm{PS}}\left(B_{U}\left(\frac{\sqrt{T}+c}{R}-\eta\right)\right)\left(m^{\mathrm{BR}}(F)-c_{2} S_{\ell}(F) T^{-\kappa^{\prime}}\right) \\
& \leq \sum_{\gamma \in \Gamma_{T}} \varphi(x \gamma) \\
& \leq \mu_{\Psi(x) \Gamma}^{\mathrm{PS}}\left(B_{U}\left(\frac{\sqrt{T}+c}{r}+\eta\right)\right)\left(m^{\mathrm{BR}}(F)+c_{2} S_{\ell}(F) T^{-\kappa^{\prime}}\right) .
\end{aligned}
$$

We now need to express $m^{\mathrm{BR}}(F)$ and $S_{\ell}(F)$ in terms of $\varphi$, and to compare the PS measures of the balls arising in (42) and (43).

Let $y \in \operatorname{supp} \varphi$. Note that, by definition of $r$ and $R, r \leq x \star y \leq R$. Hence, we may use Lemma 2.9 to deduce that for $r_{-}:=R$ and $r_{+}:=r$, there exists $T_{2}=T_{2}(x, \Omega)>0$ so that for all $T \geq T_{2}$, we have that 


$$
\begin{gathered}
\left|\mu_{\Psi(x) \Gamma}^{\mathrm{PS}}\left(B_{U}\left(\frac{\sqrt{T} \pm c}{r_{ \pm}} \pm \eta\right)\right)-\mu_{\Psi(x) \Gamma}^{\mathrm{PS}}\left(B_{U}\left(\frac{\sqrt{T}}{x \star y}\right)\right)\right| \\
\ll_{\Gamma, x}\left(\eta+\frac{c+1}{\sqrt{T}}\right)^{\alpha} \mu_{\Psi(x) \Gamma}^{\mathrm{PS}}\left(B_{U}\left(\frac{\sqrt{T}}{x \star y}\right)\right)
\end{gathered}
$$

According to Lemma 3.4, we have

$$
m^{\mathrm{BR}}(F)=\int_{P} \varphi\left(\pi_{U}(p)\right) d \nu(p) .
$$

Combining the above with (42) and (43) implies that, for some $c_{0}=c_{0}(\Gamma, x)$,

$$
\begin{aligned}
& \left(1-c_{0}\left(\eta+\frac{c+1}{\sqrt{T}}\right)^{\alpha}\right)\left(\int_{P} \varphi\left(\pi_{U}(p)\right) d \nu(p)-c_{2} S_{\ell}(F) T^{-\kappa^{\prime}}\right) \\
& \leq \frac{1}{\mu_{\Psi(x) \Gamma}^{\mathrm{PS}}\left(B_{U}\left(\frac{\sqrt{T}}{x \star y}\right)\right)} \sum_{\gamma \in \Gamma_{T}} \varphi(x \gamma) \\
& \leq\left(1+c_{0}\left(\eta+\frac{c+1}{\sqrt{T}}\right)^{\alpha}\right)\left(\int_{P} \varphi\left(\pi_{U}(p)\right) d \nu(p)+c_{2} S_{\ell}(F) T^{-\kappa^{\prime}}\right) .
\end{aligned}
$$

We are left to find $S_{\ell}(F)$. Since $B \mapsto B \Gamma$ is injective and $f$ is supported on $B$ (recall that $f$ is defined as in Lemma 3.3), using Lemma 2.10(1), Lemma 2.12, and (41), we have

$$
\begin{aligned}
S_{\ell}(F) & =S_{\ell}(f) \\
& \ll_{n} S_{\ell}(\psi) S_{\ell}\left(\varphi \circ \pi_{U}\right) \\
& \ll_{n, \Gamma} \eta^{-\ell+n-1} S_{\ell}(\varphi) .
\end{aligned}
$$

Finally, we need to put this all together. Combining (44) and (45), for any $y \in \operatorname{supp} \varphi$, we obtain that

$$
\begin{aligned}
& \left|\frac{1}{\mu_{\Psi(x) \Gamma}^{\mathrm{PS}}\left(B_{U}\left(\frac{\sqrt{T}}{x \star y}\right)\right)} \sum_{\gamma \in \Gamma_{T}} \varphi(x \gamma)-\int_{P} \varphi\left(\pi_{U}(p)\right) d \nu(p)\right| \\
& \quad \ll_{\Gamma, x}\left(\eta+\frac{c+1}{\sqrt{T}}\right)^{\alpha} \int_{P} \varphi\left(\pi_{U}(p)\right) d \nu(p)+\eta^{-\ell+n-1} S_{\ell}(\varphi) T^{-\kappa^{\prime}} \\
& \quad \ll_{\Gamma, \Omega, x}\left[\left(\eta+T^{-1 / 2}\right)^{\alpha}+\eta^{-\ell+n-1} T^{-\kappa^{\prime}}\right] S_{\ell}(\varphi) .
\end{aligned}
$$

Choose $\rho$ sufficiently small so that

$$
(\ell-n+1) \rho<\kappa^{\prime} / 2 .
$$

Let $\eta=T^{-\rho}$, for $T \geq T_{0}(x, \Omega):=\max \left\{T_{1}, T_{2}\right\}$. Let

$$
\kappa=\min \left\{\rho \alpha, \alpha / 2, \kappa^{\prime} / 2\right\} .
$$

Then we conclude that

$$
\begin{aligned}
& \left|\frac{1}{\mu_{\Psi(x) \Gamma}^{\mathrm{PS}}\left(B_{U}\left(\frac{\sqrt{T}}{x \star y}\right)\right)} \sum_{\gamma \in \Gamma_{T}} \varphi(x \gamma)-\int_{P} \varphi\left(\pi_{U}(p)\right) d \nu(p)\right| \\
& \ll \Gamma, \Omega, x T^{-\kappa} S_{\ell}(\varphi) .
\end{aligned}
$$




\section{Proof of Theorem 1.9}

In this section, we will use a partition of unity argument and the previous section to establish Theorem 1.9, which is restated below for convenience.

Theorem 6.1 Let $\Gamma$ satisfy property A. There exists $\ell=\ell(\Gamma) \in \mathbb{N}$ so that for any $0<\varepsilon<1$, there exists $\kappa=\kappa(\Gamma, \varepsilon)$ satisfying: for every $\varphi \in C_{c}^{\infty}(U \backslash G)$ and for every $x \in U \backslash G$ such that $\Psi(x) \Gamma$ is $\varepsilon$-Diophantine, and for all $T \gg_{\Gamma \text {, supp } \varphi, x} 1$,

$$
\begin{gathered}
\left|\frac{\sum_{\gamma \in \Gamma_{T}} \varphi(x \gamma)}{\mid \int_{P} \mu_{\Psi(x) \Gamma}^{\mathrm{PS}}\left(B_{U}\left(\frac{\sqrt{T}}{x \star \pi_{U}(p)}\right)\right) \varphi\left(\pi_{U}(p)\right) d \nu(p)}-1\right| \\
\ll_{\Gamma, \operatorname{supp} \varphi, x} T^{-\kappa}\left(1+S_{\ell}(\varphi) \nu\left(\varphi \circ \pi_{U}\right)^{-1}\right) .
\end{gathered}
$$

Assume throughout this section that $\Gamma$ satisfies property A. We begin by interpreting (46) in another form, as in the following lemma. This form will be easier to work with when using a partition of unity. Note that the main idea here is that for $\varphi$ of small support and for any $y \in \operatorname{supp} \varphi, x \star y$ is very close to both $R$ and $r$.

For $H \subseteq U \backslash G$ compact and $x \in U \backslash G$, define

$$
R_{H}=\max _{y \in H} x \star y \quad \text { and } r_{H}=\min _{y \in H} x \star y .
$$

Lemma 6.2 There exists $\ell=\ell(\Gamma)>0$ which satisfies the following. Let $\Omega \subseteq G$ be a compact set, let $x \in U \backslash G$ be such that $\Psi(x) \Gamma$ is $\varepsilon$-Diophantine, let $\varphi \in C_{c}^{\infty}(U \backslash G)$ with $\Psi(\operatorname{supp} \varphi) \subset \Omega$, and let $\eta>0$ be smaller than the injectivity radius of $\Omega$. Let $R=R_{\pi_{U}(\Omega)}$ and $r=r_{\pi_{U}(\Omega)}$ and assume they satisfy $\frac{R}{r}-1<\eta$. Then for $T \gg_{\Gamma, \Omega, x} 1$,

$$
\begin{aligned}
& \left|\sum_{\gamma \in \Gamma_{T}} \varphi(x \gamma)-\int_{P} \mu_{\Psi(x) \Gamma}^{\mathrm{PS}}\left(B_{U}\left(\frac{\sqrt{T}}{x \star \pi_{U}(p)}\right)\right) \varphi\left(\pi_{U}(p)\right) d \nu(p)\right| \\
& \ll \Gamma, \Omega, x \mu_{\Psi(x) \Gamma}^{\mathrm{PS}}\left(B_{U}\left(\frac{\sqrt{T}}{r}\right)\right)\left(\eta+T^{-1 / 2}\right)^{\alpha} \int_{P} \varphi\left(\pi_{U}(p)\right) d \nu(p) \\
& \quad+\mu_{\Psi(x) \Gamma}^{\mathrm{PS}}\left(B_{U}\left(\frac{\sqrt{T}}{r}\right)\right) \eta^{-\ell+(n-1) / 2} S_{\ell}(\varphi) T^{-\kappa^{\prime}} .
\end{aligned}
$$

Proof Following the arguments in the proof of Theorem 5.1 (more explicitly, the computations leading to (46)), one may deduce that there exists $\ell=\ell(\Gamma)>0$ such that for any $T \gg_{\Gamma, \Omega, x} 1$,

$$
\begin{gathered}
\left|\frac{1}{\mu_{\Psi(x) \Gamma}^{\mathrm{PS}}\left(B_{U}\left(\frac{\sqrt{T}}{x \star y}\right)\right)} \sum_{\gamma \in \Gamma_{T}} \varphi(x \gamma)-\int_{P} \varphi\left(\pi_{U}(p)\right) d \nu(p)\right| \\
\ll_{\Gamma, \Omega, x}\left[\left(\eta+T^{-1 / 2}\right)^{\alpha}+\eta^{-\ell+(n-1) / 2} T^{-\kappa^{\prime}}\right] S_{\ell}(\varphi) .
\end{gathered}
$$

Therefore, we may conclude

$$
-\mu_{\Psi(x) \Gamma}^{\mathrm{PS}}\left(B_{U}\left(\frac{\sqrt{T}}{R}\right)\right)\left[\left(\eta+T^{-1 / 2}\right)^{\alpha} \int_{P} \varphi\left(\pi_{U}(p)\right) d \nu(p)-\eta^{-\ell+(n-1) / 2} S_{\ell}(\varphi) T^{-\kappa^{\prime}}\right]
$$




$$
\begin{aligned}
& \ll_{\Gamma, \operatorname{supp} \varphi, x} \sum_{\gamma \in \Gamma_{T}} \varphi(x \gamma)-\mu_{\Psi(x) \Gamma}^{\mathrm{PS}}\left(B_{U}\left(\frac{\sqrt{T}}{R}\right)\right) \int_{P} \varphi\left(\pi_{U}(p)\right) d \nu(p) \\
& \leq \sum_{\gamma \in \Gamma_{T}} \varphi(x \gamma)-\int_{P} \mu_{\Psi(x) \Gamma}^{\mathrm{PS}}\left(B_{U}\left(\frac{\sqrt{T}}{x \star \pi_{U}(p)}\right)\right) \varphi\left(\pi_{U}(p)\right) d \nu(p) \\
& \leq \sum_{\gamma \in \Gamma_{T}} \varphi(x \gamma)-\mu_{\Psi(x) \Gamma}^{\mathrm{PS}}\left(B_{U}\left(\frac{\sqrt{T}}{r}\right)\right) \int_{P} \varphi\left(\pi_{U}(p)\right) d \nu(p) \\
& \ll \Gamma, \operatorname{supp} \varphi, x \mu_{\Psi(x) \Gamma}^{\mathrm{PS}}\left(B_{U}\left(\frac{\sqrt{T}}{r}\right)\right) \\
& \times\left[\left(\eta+T^{-1 / 2}\right)^{\alpha} \int_{P} \varphi\left(\pi_{U}(p)\right) d \nu(p)+\eta^{-\ell+(n-1) / 2} S_{\ell}(\varphi) T^{-\kappa^{\prime}}\right] .
\end{aligned}
$$

\section{Proof of Theorem 1.9 Step 1: Use an appropriate partition of $\varphi$.}

Let $\ell^{\prime}=\ell^{\prime}(\Gamma)>0$ satisfy the conclusion of Lemma 6.2 and $\ell>\ell^{\prime}$ satisfy the conclusion of Corollary 3.6 for $\ell^{\prime}$.

By Corollary 3.6, there exists a partition $\varphi_{1}, \ldots, \varphi_{k}$ of $\varphi$ satisfying Lemma 6.2 with $\Omega=\Psi(\operatorname{supp} \varphi)$ and

$$
\sum_{i=1}^{k} S_{\ell^{\prime}}\left(\varphi_{i}\right) \ll, \operatorname{supp} \varphi \eta^{-\ell+n(n+1) / 4} S_{\ell}(\varphi) .
$$

Thus, by Lemma 6.2, we have that for each $\varphi_{i}$,

$$
\begin{aligned}
\left|\sum_{\gamma \in \Gamma_{T}} \varphi_{i}(x \gamma)-\int_{P} \mu_{\Psi(x) \Gamma}^{\mathrm{PS}}\left(B_{U}\left(\frac{\sqrt{T}}{x \star \pi_{U}(p)}\right)\right) \varphi_{i}\left(\pi_{U}(p)\right) d \nu(p)\right| \\
\quad \ll \Gamma, \operatorname{supp} \varphi, x \mu_{\Psi(x) \Gamma}^{\mathrm{PS}}\left(B_{U}\left(\frac{\sqrt{T}}{r_{i}}\right)\right) . \\
\quad\left[\left(\eta+T^{-1 / 2}\right)^{\alpha} \int_{P} \varphi_{i}\left(\pi_{U}(p)\right) d \nu(p)+\eta^{-\ell+(n-1) / 2} S_{\ell}\left(\varphi_{i}\right) T^{-\kappa^{\prime}}\right] .
\end{aligned}
$$

Let

$$
r=\min \left\{r_{1}, \ldots, r_{k}\right\} .
$$

Summing over $i$, using (47), and noting that $\eta<1$ yields

$$
\begin{aligned}
& \left|\sum_{\gamma \in \Gamma_{T}} \varphi(x \gamma)-\int_{P} \mu_{\Psi(x) \Gamma}^{\mathrm{PS}}\left(B_{U}\left(\frac{\sqrt{T}}{x \star \pi_{U}(p)}\right)\right) \varphi\left(\pi_{U}(p)\right) d \nu(p)\right| \\
& \ll \Gamma, \operatorname{supp} \varphi, x \\
& \mu_{\Psi(x) \Gamma}^{\mathrm{PS}}\left(B_{U}\left(\frac{\sqrt{T}}{r}\right)\right)\left(\eta+T^{-1 / 2}\right)^{\alpha} \int_{P} \varphi\left(\pi_{U}(p)\right) d \nu(p) \\
& +\mu_{\Psi(x) \Gamma}^{\mathrm{PS}}\left(B_{U}\left(\frac{\sqrt{T}}{r}\right)\right) \eta^{-2 \ell+\left(n^{2}+3 n-2\right) / 4} S_{\ell}(\varphi) T^{-\kappa^{\prime}} .
\end{aligned}
$$




\section{Step 2: Putting it together.}

Recall

$$
I(\varphi, T, x):=\int_{P} \mu_{\Psi(x) \Gamma}^{\mathrm{PS}}\left(B_{U}\left(\frac{\sqrt{T}}{x \star \pi_{U}(p)}\right)\right) \varphi\left(\pi_{U}(p)\right) d \nu(p) .
$$

Let

$$
R=R_{\varphi}:=\max _{y \in \operatorname{supp} \varphi} x \star y .
$$

By Lemma 2.4, we have that there exists $\sigma=\sigma(\Gamma)>0$ so that

$$
\begin{aligned}
\frac{\mu_{\Psi(x) \Gamma}^{\mathrm{PS}}\left(B_{U}\left(\frac{\sqrt{T}}{r}\right)\right)}{I(\varphi, T, x)} & \leq \frac{\mu_{\Psi(x) \Gamma}^{\mathrm{PS}}\left(B_{U}\left(\frac{\sqrt{T}}{r}\right)\right)}{\mu_{\Psi(x) \Gamma}^{\mathrm{PS}}\left(B_{U}\left(\frac{\sqrt{T}}{R}\right)\right) v\left(\varphi \circ \pi_{U}\right)} \\
& \ll \Gamma\left(\frac{R}{r}\right)^{\sigma} v\left(\varphi \circ \pi_{U}\right)^{-1} \\
& \ll \Gamma, \operatorname{supp} \varphi, x v\left(\varphi \circ \pi_{U}\right)^{-1},
\end{aligned}
$$

where the last line follows because $(R / r)^{\sigma}$ is simply a constant depending on $\operatorname{supp} \varphi, \Gamma$, and $x$.

From (49) and (50), we obtain that

$$
\begin{aligned}
\left|\frac{\sum_{\gamma \in \Gamma_{T}} \varphi(x \gamma)}{I(\varphi, T, x)}-1\right| & \ll \Gamma, \operatorname{supp} \varphi, x \frac{\mu_{\Psi(x) \Gamma}^{\mathrm{PS}}\left(B_{U}\left(\frac{\sqrt{T}}{r}\right)\right)}{I(\varphi, T, x)}\left(\eta+T^{-1 / 2}\right)^{\alpha} \int_{P} \varphi\left(\pi_{U}(p)\right) d \nu(p) \\
& +\frac{\mu_{\Psi(x) \Gamma}^{\mathrm{PS}}\left(B_{U}\left(\frac{\sqrt{T}}{r}\right)\right)}{I(\varphi, T, x)} \eta^{-2 \ell+\left(n^{2}+3 n-2\right) / 4} S_{\ell}(\varphi) T^{-\kappa^{\prime}} \\
& \ll \Gamma, \operatorname{supp} \varphi, x \\
& \ll \Gamma, c \operatorname{supp} \varphi, x \\
& \ll \Gamma, \operatorname{supp} \varphi, x \\
& \left.\ll T^{-1 / 2}\right)^{\alpha}+v\left(\varphi \circ \pi_{U}\right)^{-1} \eta^{-2 \ell+\left(n^{2}+3 n-2\right) / 4} S_{\ell}(\varphi) T^{-\kappa^{\prime}}
\end{aligned}
$$

where (51) follows by choosing $\eta=T^{-\rho}$, where $\rho=1$ if $2 \ell-\frac{n^{2}+3 n-2}{4}<0$, and

$$
\rho=\frac{\kappa^{\prime}}{4 \ell-n+1-\frac{1}{2} n(n+1)}
$$

otherwise, and letting

$$
\kappa=\min \left\{\rho \alpha, \alpha / 2, \kappa^{\prime} / 2\right\}
$$

Remark 6.3 Note that the implied dependence on $x$ is quite explicit. It arises from suppressing the factors $R_{\varphi}, r_{\varphi},\left\|\Psi(x)^{-1}\right\|$, and $c$ throughout the argument. Specifically, $c$ is suppressed in the use of Lemma 6.2, and $r_{\varphi}, R_{\varphi}$ are suppressed in (51). Note that these constants depend on $x$ and $\operatorname{supp} \varphi$ through the $\star$ operation, as can be seen from the definitions and the proof of Lemma 3.2, and they can also be computed explicitly if desired. The factor of $\left\|\Psi(x)^{-1}\right\|$ is suppressed in the construction of the partition in Corollary 3.6. The implied constant from 
Theorem 2.15 also depends on $x$ through the explicit Diophantine behaviour of $x$, i.e. the $\left(\varepsilon, s_{0}\right)$.

Remark 6.4 The suppressed constants $R_{\varphi}, r_{\varphi}, c$, and $\left\|\Psi(x)^{-1}\right\|$ mentioned in Remark 6.3 are continuous functions of $x$ by definition of $\star$. This will be used in the next section.

\section{Applications}

Let $V$ be a manifold on which $G$ acts smoothly and transitively from the right, so that $V$ may be identified with $H \backslash G$ for some closed subgroup $H$ of $G$ that is the stabilizer of a point $v_{0} \in V$. Let $\sigma: H \backslash G \rightarrow V$ be the identification

$$
\sigma(H g)=v_{0} \cdot g
$$

Note that $\sigma$ is smooth because $G$ acts smoothly.

Assume further that $U \subseteq H \subseteq U M$. In particular, $\pi_{U}(H)$ is compact in $U \backslash G$ (recall from Sect. 2 that $\pi_{U}: G \rightarrow U \backslash G$ is the quotient map). Define $\theta: U \backslash G \rightarrow H \backslash G$ by

$$
\theta(U g)=H g .
$$

We will now show that $\theta$ is smooth. Since $U$ is closed, $\pi_{U}: G \rightarrow U \backslash G$ is a smooth submersion. Thus, $\theta$ is smooth if and only if $\theta \circ \pi_{U}$ is smooth. Since $\theta \circ \pi_{U}=\pi_{H}$, the quotient map from $G \rightarrow H \backslash G$, it is smooth, which establishes the smoothness of $\theta$.

For $v, u \in V$, let $x, y \in U \backslash G$ be such that $u=\sigma(\theta(x)), v=\sigma(\theta(y))$. We may define

$$
v \star u=x \star y .
$$

This is well-defined because $U M$ stabilizes $E_{1, n+1}$, and $H \subseteq U M$ (see (1) for the definition of $\star$ on $U \backslash G)$.

Recall the definition of $\Psi: U \backslash G \rightarrow G$ from Sect. 3:

$$
\Psi(U g)=a k,
$$

where $g=u a k$ is the Iwasawa decomposition of $g$.

Definition 7.1 A vector $v \in V$ is called $\varepsilon$-Diophantine if there exists $x \in U \backslash G$ such that $v=v_{0} \cdot x$ and $\Psi(x) \Gamma$ is $\varepsilon$-Diophantine. Such $x$ is called an $\varepsilon$-Diophantine representative of $v$.

Remark 7.2 Note that for any $g \in G, g^{-} \in \Lambda_{r}(\Gamma)$ if and only if $(u m g)^{-} \in \Lambda_{r}(\Gamma)$ for all um $\in U M$, since $U M$ does not change $g^{-}$. Thus, for $v \in V$, we may define the notation

$$
v^{-} \in \Lambda_{r}(\Gamma)
$$

if for any representative $\Psi(x), \Psi(x)^{-} \in \Lambda_{r}(\Gamma)$. Note also that since $\mathcal{C}_{0}$ is $M$ invariant and $A$ commutes with $M$, the definition of $v$ being $\varepsilon$-Diophantine is independent of the choice of a representative $x \in U \backslash G$.

Observe that $v$ uniquely defines a measure on $U \backslash G$ by $v\left(\varphi \circ \pi_{U}\right)$ for any continuous function $\varphi$ defined on $U \backslash G$. One can use the push-forward of this measure to $H \backslash G$ and the identification of $V$ with $H \backslash G$ to uniquely define a measure on $V$. Denote this measure by $\bar{v}$. 
Corollary 7.3 For any $0<\varepsilon<1$, there exist $\ell=\ell(\Gamma) \in \mathbb{N}$ and $\kappa=\kappa(\Gamma, \varepsilon)$ satisfying: for every $\bar{\varphi} \in C_{c}^{\infty}(V)$ and $\varepsilon$-Diophantine $v \in V$ with Diophantine representative $x \in U \backslash G$ (i.e., $v_{0} x=v$ ), and $T \gg_{\Gamma, \operatorname{supp} \bar{\varphi}, v} 1$,

$$
\left|\frac{\sum_{\gamma \in \Gamma_{T}} \bar{\varphi}(v \gamma)}{\int_{P} \mu_{\Psi(x) \Gamma}^{\mathrm{PS}}\left(B_{U}\left(\frac{\sqrt{T}}{v \star u}\right)\right) \bar{\varphi}(u) d \bar{v}(u)}-1\right| \ll_{\Gamma, \operatorname{supp} \bar{\varphi}, x} T^{-\kappa}\left(1+S_{\ell}(\bar{\varphi}) v(\bar{\varphi})^{-1}\right) .
$$

Proof Let $\ell^{\prime}$ satisfy the conclusion of Theorem 1.9 and $\ell$ satisfy the conclusion of Lemma 2.12 for $\ell^{\prime}$.

Recall the definitions of $\sigma: H \backslash G \rightarrow V$ in (52) and $\theta: U \backslash G \rightarrow H \backslash G$ in (53). Define $\varphi \in C_{c}^{\infty}(U \backslash G)$ by

$$
\varphi=\bar{\varphi} \circ \sigma \circ \theta .
$$

Let $x \in U \backslash G$ be an $\varepsilon$-Diophantine representative of $v$. In particular, note that $\sigma(\theta(x))=$ $\sigma(H \Psi(x))=v$. Then, since

$$
\varphi(x \gamma)=\bar{\varphi}(\sigma(\theta(x)) \cdot \gamma)=\bar{\varphi}(v \cdot \gamma),
$$

by Theorem 1.9, for $T \gg_{\Gamma, \operatorname{supp} \bar{\varphi}, \varepsilon, x} 1$,

$$
\begin{aligned}
& T^{-\kappa}\left(1+S_{\ell}(\varphi) v\left(\varphi \circ \pi_{U}\right)^{-1}\right) \\
& \gg_{\Gamma, \operatorname{supp} \varphi, x} T^{-\kappa}\left|\frac{\sum_{\gamma \in \Gamma_{T}} \varphi(x \gamma)}{\int_{P} \mu_{\Psi(x) \Gamma}^{\mathrm{PS}}\left(B_{U}\left(\frac{\sqrt{T}}{x \star \pi_{U}(p)}\right)\right) \varphi\left(\pi_{U}(p)\right) d v(p)}-1\right| \\
& \gg_{\Gamma, \operatorname{supp} \bar{\varphi}, x} T^{-\kappa}\left|\frac{\sum_{\gamma \in \Gamma_{T}} \bar{\varphi}(v \gamma)}{\int_{P} \mu_{\Psi(x) \Gamma}^{\mathrm{PS}}\left(B_{U}\left(\frac{\sqrt{T}}{v \star u}\right)\right) \bar{\varphi}(u) d \bar{\nu}(u)}-1\right| .
\end{aligned}
$$

Note that the dependence of $T$ on $x$ is through $\varepsilon, s_{0}$ such that $x$ is $\left(\varepsilon, s_{0}\right)$-Diophantine, and by Remark 7.2, this is in fact independent of the choice of Diophantine representative $x$ of $v$. By Remark 6.4, the dependence on $x$ in the implied constant in the above inequality can be made uniform over all representatives of $v$, as they vary by elements in $M$, a compact set. Thus, both dependencies on $x$ can be replaced by dependence on $v$.

Observe that $\varphi$ can be viewed as a function on $U \backslash H \times H \backslash G \cong U \backslash G$ by

$$
\varphi(y, x)=\operatorname{id}_{U \backslash H}(y) \cdot(\bar{\varphi} \circ \sigma)(x) .
$$

Therefore, Lemmas 2.10 and 2.12 imply

$$
S_{\ell^{\prime}}(\varphi) \ll_{H} S_{\ell^{\prime}}\left(\operatorname{id}_{U \backslash H}\right) S_{\ell^{\prime}}(\bar{\varphi} \circ \sigma) \ll_{H, \sigma, \operatorname{supp} \varphi} S_{\ell}(\bar{\varphi}),
$$

where the Sobolev norm of $\operatorname{id}_{U \backslash H}$ is finite since we are assuming $U \backslash H$ is compact.

In a similar way, one may deduce the following from Corollary 1.3 (see Remark 7.2 for the notation $\left.v^{-} \in \Lambda_{r}(\Gamma)\right)$ :

Corollary 7.4 Assume that $\Gamma$ is convex cocompact. For any $\bar{\varphi} \in C_{c}(V)$ and every $v \in V$ with $v^{-} \in \Lambda_{r}(\Gamma)$, as $T \rightarrow \infty$,

$$
\frac{1}{T^{\delta_{\Gamma} / 2}} \sum_{\gamma \in \Gamma_{T}} \bar{\varphi}(v \gamma) \asymp \int_{P} \frac{\bar{\varphi}(u)}{(v \star u)^{\delta_{\Gamma}}} d \bar{\nu}(u),
$$

where the implied constant depends on $v$ and $\Gamma$. 


\subsection{Identification with null vectors}

Let $G$ act on $\mathbb{R}^{n+1}$ by right matrix multiplication, and let

$$
V=\mathbf{e}_{n+1} G \backslash\{0\} .
$$

To better understand the set $V$, note that the representation of $\operatorname{SO}(n, 1)$ we are using is

$$
\mathrm{SO}(n, 1)=\left\{A \in \mathrm{SL}_{n+1}(\mathbb{R}): A J A^{T}=J\right\},
$$

where

$$
J=\left(\begin{array}{ccc}
0 & 0 & 1 \\
0 & -I_{n-1} & 0 \\
1 & 0 & 0
\end{array}\right)
$$

Let $P$ be such that

$$
J^{\prime}:=\left(\begin{array}{cc}
-I_{n} & 0 \\
0 & 1
\end{array}\right)=P J P^{T} .
$$

Then $V P$ is the upper half of the "light cone" in the standard representation of $\operatorname{SO}(n, 1)$. In particular, this consists of null vectors of

$$
Q^{\prime}\left(x_{1}, \ldots, x_{n+1}\right)=x_{n+1}^{2}-x_{1}^{2}-\cdots-x_{n}^{2}
$$

with $x_{n+1}>0$. In our case, $V$ consists of null vectors of

$$
Q\left(x_{1}, \ldots, x_{n+1}\right)=2 x_{1} x_{n+1}-x_{2}^{2}-\cdots-x_{n}^{2} .
$$

Proposition 7.5 Let $\Gamma$ be convex cocompact. For any $\bar{\varphi} \in C_{c}(V)$ and every $v \in V$ with $v^{-} \in \Lambda_{r}(\Gamma)$, as $T \rightarrow \infty$, we have that

$$
\frac{1}{T^{\delta_{\Gamma} / 2}} \sum_{\gamma \in \Gamma_{T}} \bar{\varphi}(v \gamma) \asymp \int_{V} \bar{\varphi}(u) \frac{d \bar{\nu}(u)}{\left(\|v\|_{2}\|u\|_{2}\right)^{\delta_{\Gamma} / 2}},
$$

where the implied constant depends on $v$ and $\Gamma$.

The measure $\bar{v}$ is described more explicitly in (59), below.

Let

$$
\mathbf{e}_{n+1}=(0, \ldots, 0,1) \in \mathbb{R}^{n+1}
$$

Then

$$
\operatorname{Stab}_{G}\left(\mathbf{e}_{n+1}\right)=U M,
$$

and hence

$$
A \times M \backslash K \cong U M \backslash G \cong V
$$

via right matrix multiplication

$$
U M g \mapsto \mathbf{e}_{n+1} g .
$$

We will now interpret Corollary 7.3 in this setting. We start by understanding the measure $\bar{v}$.

We view $V$ as $(M \backslash K) \times \mathbb{R}^{+}$, via the "polar decomposition" of $v \in V$,

$$
v=\|v\|_{2} \mathbf{e}_{n+1} k=\mathbf{e}_{n+1} a_{-\log \|v\|_{2}} k,
$$


where $\mathbb{R}^{+}=\{r \in \mathbb{R}: r>0\}$ and $\|\cdot\|_{2}$ denotes the Euclidean norm on $V$. We may also identify $M \backslash K$ with $\partial\left(\mathbb{H}^{n}\right)$ via

$$
M k \mapsto w_{o}^{-} k .
$$

Thus, given $v \in V$, (56) and (57) uniquely determine a pair $\left(a_{-} \log \|v\|_{2}, M k\right) \in A \times M \backslash K$, or equivalently, a pair $\left(a_{-} \log \|v\|_{2}, w_{o}^{-} k\right) \in A \times \partial\left(\mathbb{H}^{n}\right)$.

Viewing $\partial\left(\mathbb{H}^{n}\right)$ as $M \backslash K$ as in (57), we may in turn identify this with $\mathbb{S}^{n} \subseteq \mathbb{R}^{n+1}$ via

$$
w_{o}^{-} k \mapsto \mathbf{e}_{n+1} k .
$$

Thus, $v_{o}$ uniquely determines a measure $\bar{v}_{o}$ on $\mathbb{S}^{n} \cap V$ via

$$
d \bar{v}_{o}\left(\mathbf{e}_{n+1} k\right)=d v_{o}\left(w_{o}^{-} k\right) .
$$

Then, since $K$ stabilizes $o$ and $M$ stabilizes $w_{o}, \bar{v}$ can be described from (13): if $s=$ $\beta_{\left(a_{-\log \|v\|_{2}} k\right)^{-}}\left(o, a_{-\log \|v\|_{2}} k(o)\right)=\log \|v\|_{2}$,

$$
\begin{aligned}
& d \bar{\nu}(v):=d v\left(a_{-} \log \|v\|_{2} k\right) \\
& =e^{\delta_{\Gamma} \beta_{\left(a_{-} \log \|v\|_{2} k\right)^{-}}\left(o, a_{-} \log \|v\|_{2} k(o)\right)} d v_{o}\left(w_{o}^{-} a_{-} \log \|v\|_{2} k\right) d s \\
& =e^{\delta_{\Gamma} s} d v_{o}\left(w_{o}^{-} k\right) d s \\
& =\|v\|_{2}^{\delta_{\Gamma}-1} d \bar{v}_{o}\left(\mathbf{e}_{n+1} k\right) d\|v\|_{2} \text {. }
\end{aligned}
$$

For $v \in V$, define

$$
v^{-}:=\mathbf{e}_{n+1} k \in \mathbb{S}^{n},
$$

where $v$ corresponds to $\left(a_{-} \log \|v\|_{2}, M k\right) \in A \times M \backslash K$. Then we have

$$
d \bar{\nu}(v)=\|v\|_{2}^{\delta_{\Gamma}^{-1}} d \bar{\nu}_{o}\left(v^{-}\right) d\|v\|_{2} .
$$

As discussed in the previous section, $v \star u$ may be computed by the formula in (1) for any choice of representatives of $v$ and $u$ in $U \backslash G$. In particular, if

$$
v=\|v\|_{2} \mathbf{e}_{n+1} k_{v}, \quad u=\|u\|_{2} \mathbf{e}_{n+1} k_{u},
$$

then

$$
v \star u=\sqrt{\frac{1}{2}\|v\|_{2}\|u\|_{2} \max _{1 \leq i, j \leq n+1}\left|\left(k_{v}^{-1}\right)_{i, 1}\left(k_{u}\right)_{n+1, j}\right|},
$$

where $k_{i, j}$ denotes the $(i, j)$ entry of $k$. In particular

$$
v \star u \asymp \sqrt{\|v\|_{2}\|u\|_{2}} .
$$

Putting this together with Corollary 7.4 yields the proposition.

\subsection{Wedge products}

The previous example can be generalized to $\bigwedge^{j} \mathbb{R}^{n+1}$ for any $1 \leq j \leq n$. Fix $j$, and let

$$
W=\bigwedge^{j} \mathbb{R}^{n+1}, \quad \text { and } \quad v_{0}=v_{0}(j)=e_{n-j+1} \wedge \cdots \wedge e_{n+1},
$$


with $G$ acting on $W$ by right multiplication. Then,

$$
\operatorname{Stab}_{e_{n-j+1} \wedge \cdots \wedge e_{n+1}}=U \cdot M_{j}
$$

for some $M_{j} \subseteq M$. Define

$$
V=v_{0} G \backslash\{0\} .
$$

Fix a norm on $V$ which is invariant under $K$ such that $\left\|v_{0}\right\|=1$.

Since any $v \in V$ can be written as

$$
v=v_{0} a_{-\log \|v\|} k,
$$

where $k \in M_{j} \backslash K$, in a similar way to the construction in the previous section, one can show that if $a_{-\log \|v\|} k \in U P$ and can be written as uamv $\in U A M \tilde{U}$, then

$$
d \bar{\nu}(v)=\|v\|^{\delta_{\Gamma}-1} d v_{o}\left(v^{-}\right) d\|v\| d m,
$$

where $v^{-}:=w_{o}^{-} k$, and $d m$ is the push forward of the probability Haar measure on $M_{j} \backslash M$. $d \bar{v}(v)$ is zero if $a_{-\log \|v\|} k \notin U P$, because the original measure $v$ is supported on $P$.

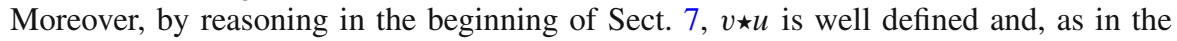
previous section, we have that

$$
v \star u \asymp \sqrt{\|v\|\|u\|} .
$$

Acknowledgements We would like to thank Barak Weiss for bringing this problem to our attention, and Amir Mohammadi for many useful discussions. We are grateful to the anonymous referee for their insightful comments on an earlier version of this manuscript, which significantly improved the paper. The first author was partially supported by the Eric and Wendy Schmidt Fund for Strategic Innovation.

Open Access This article is licensed under a Creative Commons Attribution 4.0 International License, which permits use, sharing, adaptation, distribution and reproduction in any medium or format, as long as you give appropriate credit to the original author(s) and the source, provide a link to the Creative Commons licence, and indicate if changes were made. The images or other third party material in this article are included in the article's Creative Commons licence, unless indicated otherwise in a credit line to the material. If material is not included in the article's Creative Commons licence and your intended use is not permitted by statutory regulation or exceeds the permitted use, you will need to obtain permission directly from the copyright holder. To view a copy of this licence, visit http://creativecommons.org/licenses/by/4.0/.

\section{References}

1. Aubin, T.: Nonlinear Analysis on Manifolds, vol. 252. Springer, New York (1982)

2. Bowditch, B.H.: Geometrical finiteness for hyperbolic groups. J. Funct. Anal. 113(2), 245-317 (1993)

3. Das, T., Fishman, L., Simmons, D., Urbański, M.: Extremality and dynamically defined measures, part II: Measures from conformal dynamical systems. Ergod. Theory Dyn. Syst. (2021). https://doi.org/10. 1017/etds.2020.46

4. Einsiedler, M., Ward, T.: Ergodic Theory with a View Towards Number Theory, Graduate Texts in Mathematics, vol. 259. Springer-Verlag London Ltd, London (2011)

5. Gorodnik, A.: Uniform distribution of orbits of lattices on spaces of frames. Duke Math. J. 122(3), 549-589 (2004)

6. Gorodnik, A., Nevo, A.: Ergodic theory and the duality principle on homogeneous spaces. Geom. Funct. Anal. 24, 159-244 (2014)

7. Gorodnik, A., Maucourant, F.: Proximality and equidistribution on the Furstenberg boundary. Geom. Dedicata 113, 197-213 (2005)

8. Gorodnik, A., Weiss, B.: Distribution of lattice orbits on homogeneous varieties. Geom. Func. An. 17, 58-115 (2007) 
9. Kleinbock, D., Margulis, G.A.: Bounded orbits of nonquasiunipotent flows on homogeneous spaces, Sinai's Moscow Seminar on Dynamical Systems, American Mathematical Society Translations Series, 2, 171, American Mathematical Society, Providence, RI, pp. 141-172 (1996)

10. Ledrappier, F.: Distribution des orbites des réseaux sur le plan réel. C.R. Acad. Sci. Paris Sr. I Math. 329(1), 61-64 (1999)

11. Ledrappier, F., Pollicott, M.: Distribution results for lattices in $\mathrm{SL}_{2}\left(\mathbb{Q}_{p}\right)$. Bull. Braz. Math. Soc. (N.S.) 36(2), 143-176 (2005)

12. Maucourant, F., Schapira, B.: Distribution of orbits in the plane of a finitely generated subgroup of $\operatorname{SL}(2, \mathbb{R})$. Am. J. Math. 136, 1497-1542 (2014)

13. Maucourant, F., Weiss, B.: Lattice actions on the plane revisited. Geometriae Dedicata 157, 1-21 (2012)

14. Mohammadi, A., Oh, H.: Classification of joinings for Kleinian groups. Duke Math. J. 165(11), 21552223 (2016)

15. Noguiera, A.: Orbit distribution on $\mathbb{R}^{2}$ under the natural action of $\operatorname{SL}(2, \mathbb{Z})$. Indag. Math. (N.S.) 13(1), 103-124 (2002)

16. Pollicott, M.: Rates of Convergence for Linear Actions of Cocompact Lattices on the Complex Plane, Integers, Volume 11B, Proceedings of the Leiden. Numeration Conference 2010 (2011)

17. Stratmann, B., Urbański, M.: Diophantine extremality of the Patterson measure. Math. Proc. Cambridge Philos. Soc. 140, 297-304 (2006)

18. Tamam, N., Warren, J. M.: Effective equidistribution of horospherical flows in infinite volume rank one homogeneous spaces, arXiv:2007.03135

Publisher's Note Springer Nature remains neutral with regard to jurisdictional claims in published maps and institutional affiliations. 